\title{
Disponible en \\ Predicción de fracturas en estructuras de hormigón combinando los métodos de elementos finitos y de elementos discretos
}

\author{
Fractures prediction in concrete structures combining the finite and discrete element methods \\ Francisco Zárate $^{\mathrm{a}, \mathrm{b}, *}$ y Eugenio Oñate ${ }^{\mathrm{a}, \mathrm{b}}$

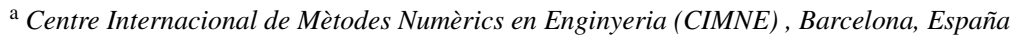 \\ ${ }^{\mathrm{b}}$ Universitat Politècnica de Catalunya (UPC), Barcelona, España \\ Recibido el 8 de enero de 2018; aceptado el 17 de mayo de 2018 \\ Disponible en Internet el 17 de julio de 2018
}

\begin{abstract}
Resumen
El presente trabajo plantea los conceptos básicos y de aplicación de la estrategia que combina los métodos de elementos finitos y de elementos discretos para el estudio de la propagación de fracturas en estructuras de hormigón. El cálculo de la estructura, modelada como un continuo, se inicia con los métodos de elementos finitos y se hace uso de los métodos de elementos discretos para iniciar y hacer crecer las grietas que puedan aparecer en la estructura. Esta metodología ha sido propuesta por los autores en 2 y 3 dimensiones. Recientemente se ha agregado el uso de elementos unidimensionales de acero embebidos en el continuo para modelar el efecto resistente del armado en estructuras de hormigón. En el trabajo se presentan diferentes ejemplos de aplicación al estudio de la rotura de piezas y estructuras de hormigón en masa y armado, así como la rotura múltiple de una estructura histórica de mampostería debida a un terremoto.
\end{abstract}

(C) 2018 Asociación Española de Ingeniería Estructural (ACHE). Publicado por Elsevier España, S.L.U. Todos los derechos reservados.

Palabras clave: Método de elementos discretos; Método de elementos finitos; Mecánica de fractura; Estrategia elementos finitos-discretos

\begin{abstract}
This paper presents the basic concepts and application of the strategy that combines finite element methods (FEM) and discrete elements (DEM) for the study of the propagation of fractures in concrete structures. The calculation of the structure, modeled as a continuum, begins with the FEM and the DEM is used to start and grow the cracks that may appear in the structure. This methodology has been proposed by the authors in two and three dimensions. Recently, the use of one-dimensional steel elements embedded in the continuum has been added to model the reinforcement in concrete structures. The work presents different examples of application to the study of the breakage of parts and structures of concrete in mass and armed, as well as the multiple breakage of a historic structure of masonry due to an earthquake.
\end{abstract}

(C) 2018 Asociación Española de Ingeniería Estructural (ACHE). Published by Elsevier España, S.L.U. All rights reserved.

Keywords: Finite element method; Discrete element method; Fracture mechanics; Finite-discrete element strategy

\section{Introducción}

Recientemente muchos autores han realizado interesantes desarrollos con la finalidad de poder definir el inicio y creci-

\footnotetext{
* Autor para correspondencia.

Correo electrónico: zarate@ cimne.upc.edu (F. Zárate).
}

miento de una fractura dentro de una estructura, modelada como un medio continuo, ya sea frágil o dúctil [1-4].

Uno de los trabajos más recientes discretiza el medio continuo por medio de elementos discretos de forma circular (en 2 dimensiones) o esférica (en 3 dimensiones) [5]. Sin embargo, la dificultad inherente para calibrar los parámetros del material en el método de los elementos discretos (DEM, por sus siglas en inglés), así como la necesidad de contar con un gran número de 
elementos discretos [2,5], pone en duda su efectividad, a pesar de que cualitativamente el número y dirección de las grietas y los resultados numéricos obtenidos de la carga última de la estructura son bastante aceptables.

Este trabajo hace uso de una estrategia que utiliza el método de elementos finitos (FEM, por sus siglas en inglés) para discretizar la estructura inicial y seguir su comportamiento bajo cargas crecientes hasta el inicio de la primera fisura. Tras ello se utiliza una técnica de eliminación de los elementos finitos dañados y se introducen elementos discretos en los labios de las fisuras [6-8]. La denominada técnica FEM-DEM se ha utilizado con éxito en 2 y 3 dimensiones $[9,10]$ para estudiar el comportamiento no lineal y el fallo de estructuras de hormigón y mampostería bajo diferentes solicitaciones cuasi-estáticas y dinámicas.

Otros aspectos importantes inherentes a la formulación FEMDEM son el uso de un campo de esfuerzos suavizado, la conservación de la masa y el uso de un algoritmo simple para asegurar el contacto posfractura entre las paredes de la grieta. Adicionalmente, la armadura de acero se considera embebida en la malla de elementos finitos, lo cual simplifica los cálculos.

\section{Formulación método de elementos finitos-método de elementos discretos}

En general la estrategia que se sigue para el cálculo no lineal de estructuras con modelos de elementos finitos de sólido bidimensionales (2D) o tridimensionales (3D) [11] pasa para evaluar a lo largo del tiempo la respuesta de la estructura discretizada con una malla de elementos finitos. En dicha malla las conectividades interelementales emulan los enlaces entre los elementos que se degradan de forma progresiva, generalmente mediante un sencillo modelo de daño isótropo. Dichos enlaces interelementales pueden interpretarse como las conexiones entre una colección de elementos discretos que reemplazarían los nodos de la malla de elementos finitos. Esta analogía es el punto de partida de la técnica FEM-DEM $[9,10]$.

La estrategia FEM-DEM se puede resumir en los 5 pasos siguientes:

- Discretización del continuo que modela la estructura mediante el FEM.

- Obtención del campo de tensiones sobre la estructura.

- Obtención del daño en el interior y los lados de los elementos.

- Discretización mediante el DEM.

- Integración temporal en subpasos.

Debido a la naturaleza de los ejemplos se describirán los pasos anteriores enfocados al caso 3D, utilizando tetraedros lineales de 4 nodos para la discretización con el FEM.

Una de las claves de la técnica FEM-DEM es el procedimiento para pasar el daño producido en un elemento finito, con la consiguiente degradación de la rigidez elemental, a una discretización de los labios de la fisura por elementos discretos de forma circular (en 2D) o esférica (en 3D).

Cuando el daño que se induce en un elemento es mayor que un cierto valor se considera que la rigidez del elemento se encuentra tan disminuida que es posible eliminarlo; para ello se utiliza una técnica de eliminación de elementos [6-8]. En ese momento se crean nuevos elementos discretos en los vértices del tetraedro eliminado (en 3D), lo que permite la apertura de la grieta en el continuo discretizado por los elementos finitos, mientras que los labios de la fisura quedan definidos por los elementos discretos. A medida que la fisura crece, e incluso se ramifica, algunos elementos discretos pueden separarse de la malla de elementos finitos creando una disgregación del continuo. El hecho de utilizar elementos discretos para definir las grietas permite, de manera natural, considerar la apertura y cierre de estas sin añadir procedimientos adicionales.

\section{Discretización mediante el método de elementos finitos}

El FEM es probablemente la técnica numérica más popular para modelar el inicio y la propagación de fisuras en materiales friccionales (hormigón, rocas, mampostería, cerámicas, etc.) [12-16]. Sin embargo, la mayoría de los procedimientos basados en el FEM para la predicción de la aparición y evolución de grietas utilizan formulaciones de elementos muy sofisticadas, que en ocasiones requieren un remallado en la vecindad de las posibles grietas $[15,16]$. El enfoque seguido en este trabajo utiliza el FEM para modelar la estructura mediante elementos de sólido (2D o 3D), cuya eventual fractura se describe mediante el DEM. En trabajos previos de la técnica FEM-DEM hemos utilizado el sencillo triángulo de 3 nodos en 2D y el tetraedro de 4 nodos en 3D [9,10].

Considerando, por ejemplo, el caso 3D, inicialmente todo el dominio de la estructura se discretiza con una malla de tetraedros, como en cualquier análisis lineal 3D por el FEM. La no linealidad se modela introduciendo una degradación de la rigidez elemental mediante la clásica teoría de daño isótropo [13,17].

El efecto resistente de la armadura de acero se modela mediante elementos unidimensionales (1D) embebidos en la malla de tetraedros. Ello implica localizar los puntos de intersección entre los elementos 1D que modelan el acero y todos los tetraedros que discretizan el dominio. Para cada tetraedro intersectado se determinan los puntos de intersección, los cuales corresponden a los nodos del segmento de la armadura embebida en el tetraedro. Como es posible describir el desplazamiento de dichos nodos en función de los nodos del tetraedro, la matriz de rigidez del elemento $1 \mathrm{D}$ de acero es función de dichos desplazamientos.

Esta técnica no incrementa el número de ecuaciones a resolver y permite de una forma muy sencilla tomar en cuenta la rigidez asociada a la armadura.

\subsection{Definición del campo de tensiones}

El considerar un campo de tensiones adecuado es fundamental para poder definir correctamente el nivel del daño en la estructura y la trayectoria de las fisuras. Dado que en el FEM las tensiones entre elementos son discontinuas [11], es esencial realizar un alisado de las mismas para obtener un campo continuo de las tensiones entre elementos. En problemas 2D se obtiene un campo de tensiones continuo alisando los valores de 
las tensiones, calculadas inicialmente en el baricentro de cada elemento, para obtener su valor el centro de los lados de cada elemento. Esta estrategia corresponde al método de la parcela superconvergente propuesta por Zienkiewicz y Zhu [18], y evita agregar términos de estabilización al campo de tensiones como es necesario en otros procedimientos alternativos [12-14].

En análisis 3D se procede de manera similar, ya que el campo de tensiones entre elementos es discontinuo, por lo que se utiliza el mismo mecanismo de suavizado. Es decir, las tensiones en cada una de las aristas de un elemento tetraédrico es la media aritmética de las tensiones evaluadas dentro de todos los elementos que comparten dicha arista.

Al igual que en el caso 2D [9], se ha utilizado el criterio de fallo de Rankin para definir el inicio de una fractura en materiales frágiles tomando en cuenta la tensión principal mayor $\sigma_{l}[13,17]$. El crecimiento del daño se realiza mediante la siguiente ley exponencial que degrada progresivamente la rigidez del material [19].

$d=1-\frac{\sigma f}{\sigma 1} \exp \left[A\left(1-\frac{\sigma 1}{\sigma f}\right)\right] \operatorname{con} A=\left[\frac{G E_{0}}{l \sigma_{f}^{2}}-\frac{1}{2}\right]^{-1}$

donde $l$ es la longitud de la arista del elemento finito dañado, $\sigma f$ es el límite elástico a tracción del material, $G$ la energía de fractura y $E_{0}$ el módulo de Young del material no dañado. Resulta obvio que entre 2 pasos de tiempo el daño de un lado o una arista no puede disminuir, ya que ello implicaría la reparación espontánea del material, lo cual es termodinámicamente inadmisible.

\subsection{Definición del daño elemental}

Una vez que el daño ha sido evaluado en cada lado o arista de un elemento (es decir, un enlace virtual entre elementos discretos) el daño sobre el conjunto del elemento se calcula como el máximo daño existente en todos los planos que cortan un triángulo (2D) o un tetraedro (3D) (fig. 1). En cada tetraedro existen 4 planos de corte que aíslan un vértice (fig. 1 b) y otros 3 que aíslan 2 vértices (fig. 1 c). De esta manera, el daño sobre el plano de corte se define como el valor medio del daño de cada arista del elemento que corta dicho plano.

Si el daño elemental sobrepasa un cierto umbral entonces se elimina el triángulo o tetraedro de la malla [6-8] y se crean nuevos elementos discretos en los vértices. El tamaño de estos elementos discretos se define de manera que se conserve la masa del elemento eliminado.

\section{Discretización mediante método de elementos discretos}

Cuando un tetraedro es totalmente eliminado (i.e. su rigidez es despreciable) se crean 4 nuevos elementos discretos en los vértices de dicho elemento finito. La creación de dichos elementos discretos queda condicionada a que no hayan sido creados con anterioridad debido a la eliminación de algún elemento finito vecino. En este trabajo se han utilizado círculos y esferas para representar los elementos discretos en $2 \mathrm{D}$ y $3 \mathrm{D}$, respectivamente.
La masa de cada elemento discreto corresponde a la masa nodal que es compatible con la obtenida mediante elementos finitos. El radio de un nuevo elemento discreto esférico se obtiene de tal manera que sea el máximo que garantice el contacto con las esferas vecinas, pero sin crear solapamientos. Existen otros algoritmos para generar elementos discretos [2,20], sin embargo, el propuesto, a pesar de su simplicidad, ha dado buenos resultados.

Una vez que se crea un elemento discreto las fuerzas en las interfaces de contacto se usan para definir la interacción de dicho elemento con los adyacentes. Estas fuerzas se deben únicamente a un mecanismo de contacto en las direcciones normal y tangencial al plano de contacto entre esferas (en 3D), considerando el radio mínimo entre las 2 partículas en contacto [5].

En problemas 3D la fuerza normal de contacto se genera en el punto de contacto entre 2 esferas y viene dada por:

$F_{n}^{i j}=\frac{A^{i j}}{d_{i j}} E_{0} u_{n} \operatorname{con} A^{i j}=\pi r_{c}^{2}$

donde $A^{i j}$ es el área de la superficie de contacto entre las 2 esferas, $r_{c}$ es el radio de la menor de las 2 esferas que interactúan en la interfaz $i j ; u_{n}$ es el solapamiento en la dirección de los centros de las dos esferas y $d_{i j}$ es la distancia entre dichos centros $[5]$.

La fuerza tangencial $F_{s}$ en el punto de contacto se descompone en 2 direcciones ortogonales $s_{1}$ y $s_{2}$ contenidas en el plano normal a la dirección $d^{i j}$. Para cada dirección $s_{j}$ la fuerza tangencial se describe por:

$F_{S}=\min \left\{\frac{u_{s i} \frac{E_{0}}{2(1+u)}}{\mu_{n}^{F}\left(\frac{u_{s i}}{u_{s}}\right)}\right.$

donde $\mu$ es el coeficiente de rozamiento entre esferas y $u$ es el coeficiente de Poisson. Más detalles de las ecuaciones (2) y (3) se pueden encontrar en Oñate et al. [5].

En general, el número total de elementos discretos generados en un análisis es solo una pequeña fracción del número total de nodos en la malla de elementos finitos [10]. Por lo tanto, los algoritmos de búsqueda para localizar las fuerzas de contacto entre elementos discretos solo representan un pequeño porcentaje del tiempo total de cálculo.

Es importante mencionar que la estrategia del uso de elementos discretos para definir el contacto entre los labios de una fisura permite resolver problemas estructurales en donde existe apertura y cierre de múltiples grietas, como se muestra en el análisis sísmico de un edificio histórico de mampostería, presentado al final de este artículo.

\section{Integración temporal en subpasos}

Uno de los principales problemas en el análisis 3D en el tiempo es el gran número de ecuaciones que surgen de la discretización. Sumando a estos la dificultad para considerar un gran período de tiempo, se concluye que en problemas dinámicos la integración implícita en el tiempo es la mejor estrategia de solución. Sin embargo, este tipo de integraciones son complejas de utilizar en el DEM debido a que es imposible identificar y 
a

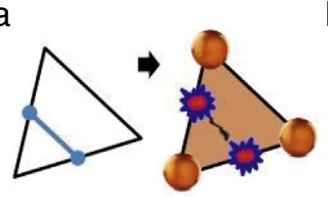

b

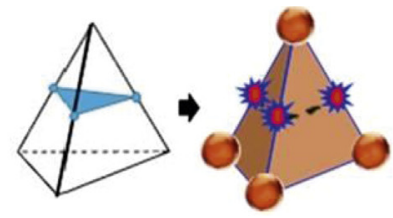

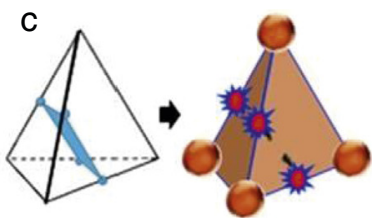

Figura 1. Planos de corte. a) Elemento triangular; b) tetraedro aislando un vértice; c) tetraedro aislando 2 vértices.

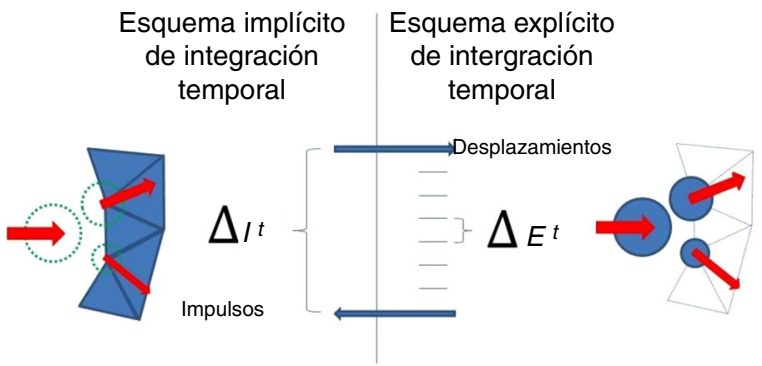

Figura 2. Integración temporal en subetapas utilizado para el análisis conjunto de elementos finitos y discretos.

cuantificar correctamente los contactos y las fuerzas de contacto entre elementos discretos.

La implementación para el estudio de problemas transitorios seguida en esta investigación corresponde a un esquema de integración temporal de subetapas en el cual los elementos finitos se calculan mediante un esquema implícito de Newmark, y los elementos discretos mediante un esquema explícito de diferencias centradas. Normalmente el incremento de tiempo del esquema implícito suele ser 100 veces mayor que el explícito.

La ventaja de esta estrategia es que el número de elementos discretos suele ser mucho menor que el de elementos finitos que discretizan la estructura, por lo que la integración explícita realizada dentro de un paso de tiempo implícito es bastante rápida. Antes de comenzar un nuevo paso de tiempo $\Delta_{i} t$ en el esquema implícito, se calcula el mismo período de tiempo con un esquema explícito sobre los elementos discretos, usando un incremento de tiempo $\Delta_{E} t$.

El contacto entre elementos discretos se cuantifica por la suma de los impulsos que se producen a lo largo del análisis explícito, y se expresa como una fuerza sobre los elementos discretos, aplicada en el tiempo $t+1$ correspondiente al esquema implícito, tal como se muestra en la figura 2.

Se ha observado que no es aconsejable que los intervalos de tiempo $\Delta_{i} t$ y $\Delta_{E} t$ tengan una relación mayor que 1: 500, ya que puede haber discrepancias entre la solución explícita y la implícita. Teniendo esto en cuenta, la estrategia de solución en subpasos implementada permite obtener excelentes resultados, como se muestra en los ejemplos del siguiente apartado.

\section{Ejemplos}

En este apartado se exponen varios ejemplos a fin de mostrar el buen comportamiento de la estrategia FEM-DEM descrita. El primer ejemplo corresponde al estudio 3D de una probeta normalizada en un ensayo a tracción. El segundo ejemplo es una viga de hormigón bientallada donde predomina la fractura

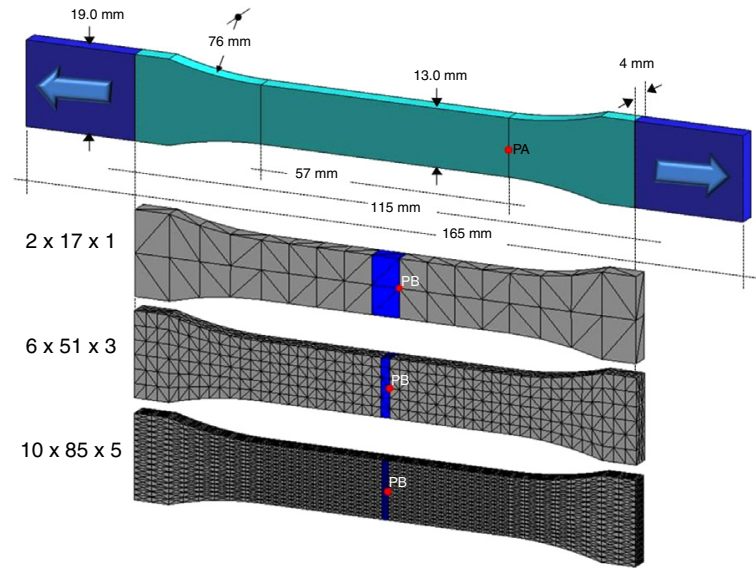

Figura 3. Ensayo normalizado 3D de tracción. Malla de elementos finitos y dimensiones según la norma ASTMD638.

en modo mixto. El tercer ejemplo corresponde al ensayo de tracción indirecta, ampliamente usado en mecánica de rocas. El cuarto ejemplo consiste en un ensayo de cortante en hormigón propuesto por Luong [21]. El quinto ejemplo es un ensayo de una probeta a compresión simple. El sexto ejemplo es el estudio de un forjado reticular de hormigón armado, afectado por un asentamiento diferencial. El acero se considera embebido y solidario con los desplazamientos de los elementos finitos utilizados.

Finalmente, en el sexto ejemplo, se presenta el análisis sísmico de la nave central de la iglesia del monasterio de Poblet, formada por elementos de mampostería, aplicándole un sismo de $6 M_{w}$. En este ejemplo se puede observar como la estrategia FEM-DEM modela el efecto de la apertura y cierre de múltiples grietas.

\subsection{Ensayo normalizado de tracción}

El primer ejemplo corresponde al análisis de fractura de una probeta de hormigón sujeta a fuerzas de tracción. El objetivo principal es mostrar la independencia del tamaño de la malla en la generación de la grieta, de manera que la energía utilizada en la fractura sea independiente del tamaño elemental. La geometría se define de acuerdo con la norma D638 de la Sección Norteamericana de la Asociación Internacional de Ensayo de Materiales (American Society for Testing and Materials) [22]. En la figura 3 se muestran las 3 mallas de elementos tetraédricos de 4 nodos utilizadas, así como las condiciones de contorno. La probeta se carga imponiendo un campo de velocidad constante de tracción en sus extremos, representados por la zona oscura en la figura. 


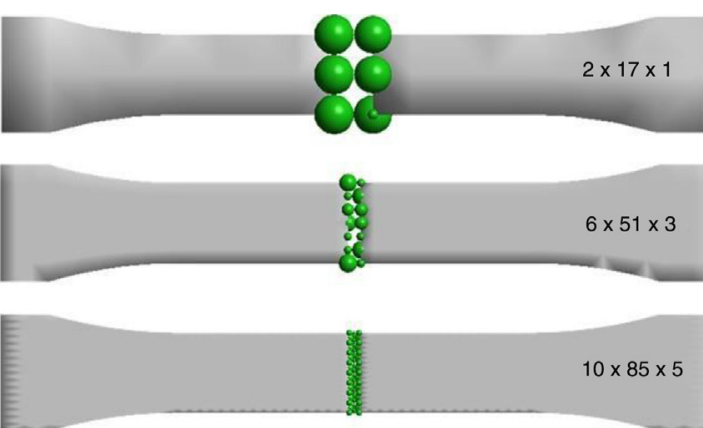

Figura 4. Ensayo normalizado de tracción. Zona agrietada incluyendo los elementos discretos generados para las 3 mallas de elementos finitos consideradas.

El estudio se ha realizado utilizando la metodología FEMDEM en 3D antes descrita. Con el fin de localizar la fractura, solo se permite que una banda de elementos se rompa al nivel de la tensión de fallo, usando el modelo de daño mencionado. Los resultados obtenidos se analizan dibujando los desplazamientos de los puntos PA y PB mostrados en la figura 3.

El módulo de Young, el coeficiente de Poisson y la densidad son respectivamente $M_{w}=30 \times 10^{9} \mathrm{~Pa}, v=0,2, y=1,0 \times 10^{3}$ $\mathrm{N} / \mathrm{m}^{3}$, la tensión máxima de tracción $\sigma_{f}=10 \times 10^{3} \mathrm{~Pa}$ y la energía de fractura $G=7,5 \times 10^{-3} \mathrm{~J} / \mathrm{m}^{2}$.

La probeta se deforma aplicando una velocidad constante de tracción de $0,5 \times 10^{-7} \mathrm{~m} / \mathrm{s}$ en ambos extremos. La figura 4 muestra la geometría dañada para las 3 mallas del FEM consideradas.

Obsérvese que cuando se produce una fractura se crean elementos discretos en los labios de la única fisura que aparece en este ejemplo, como se explica en los apartados anteriores. El tamaño de las esferas no es uniforme debido a que no todos los elementos llegan al daño máximo de manera simultánea.

Con objeto de evaluar la apertura de la grieta se analiza el desplazamiento de los puntos PA y PB situados a la derecha y en el centro de la probeta, respectivamente (fig. 3). La figura 5 muestra la relación carga-desplazamiento en estos puntos. Para las 3 mallas consideradas la evolución del desplazamiento es muy similar, y de acuerdo con los resultados esperados [9]. Debido a que los elementos por donde se abre la grieta tienen un tamaño diferente para cada malla, el desplazamiento del punto PB en la región elástica se hace más pequeño a medida que se reduce el tamaño del elemento.

\subsection{Viga bientallada a flexión}

El siguiente ejemplo corresponde al ensayo de una viga de hormigón en masa bientallada. El análisis se realiza mediante las hipótesis de tensión plana y es un buen ejemplo de fractura en modo mixto. La viga se sostiene en 2 puntos y se somete a flexión aplicando un desplazamiento impuesto mediante control de velocidad equivalente a $1 \mathrm{~mm} / \mathrm{s}$ en los 2 puntos representados en la figura 6. Igualmente, en dicha figura también se muestran la geometría y las dimensiones de la probeta.

La viga presenta 2 puntos singulares en la punta de las 2 entallas, en donde las tensiones de tracción son altas y el daño comienza en esta zona. Las propiedades del material son $E_{0}$
$=30 \times 10^{9} \mathrm{~Pa}, v=0,2, \sigma_{f}=2 \mathrm{MPa}$ y $G=1 \times 10^{2} \mathrm{~J} / \mathrm{m}^{2}$. El problema ha sido resuelto con la técnica FEM-DEM en 2D.

En la figura 7 se muestra un detalle de las 3 diferentes mallas utilizadas, formadas por 1.165 nodos y 2.202 elementos triangulares lineales para la malla gruesa, 1.847 nodos y 3.480 elementos para la malla intermedia y 5.747 nodos y 11.206 elementos para la malla fina. El análisis se ha realizado tanto de forma cuasiestática como de forma dinámica, respetando la velocidad de aplicación de la carga. En ambos casos los resultados han sido muy similares, como se observa en la figura 8 .

La figura 9 muestra la dirección de las fisuras para las 3 mallas analizadas, las cuales coinciden con los experimentos numéricos [14]. La figura 8 muestra la relación entre la reacción y el desplazamiento impuesto en cualquiera de los 2 puntos representados en la figura 6 (los resultados son idénticos para los 2 puntos). Los gráficos son concordantes con los resultados obtenidos por Cervera et al. [14].

\subsection{Ensayo de tracción indirecta}

El ensayo brasileño de tracción indirecta (Brazilian Tensile Strength) es un procedimiento sencillo para evaluar la resistencia a la tracción de hormigón y geomateriales. La probeta de hormigón analizada es un cilindro de, $2 \mathrm{~m}$ de diámetro $(D)$ y $0,1 \mathrm{~m}$ de espesor $(t)$, sujeto a una carga diametralmente opuesta (fig. 10). El valor de la resistencia a la tracción se calcula mediante la siguiente expresión [23,24]:

$\sigma_{f} \frac{2 P}{\pi t D}$

Donde $P$ es el valor de la carga aplicada. Las propiedades del material son $E_{0}=21 \times 10^{9} \mathrm{~Pa}, v=0,2, y=7,8 \times 10^{3} \mathrm{~N} / \mathrm{m}^{3}$, $\sigma_{f}=10 \mathrm{KPa}$ y $G=1 \times 10^{2} \mathrm{~J} / \mathrm{m}^{2}$, lo que proporciona una carga máxima de fallo de $P=314,16 \mathrm{~N}$.

Para realizar los análisis se han usado 3 mallas de 9.338, 31.455 y 61.623 elementos tetraédricos lineales, como se muestra en la figura 10. El ensayo se realiza imponiendo una velocidad constante vertical en la parte superior de la probeta.

La figura 11 muestra la grieta y los elementos discretos generados. Se puede observar que el patrón de fisuración es similar para las 3 mallas y de acuerdo con el resultado esperado. La figura 12 muestra la curva de carga-desplazamiento. Los valores obtenidos para la resistencia a la tracción máxima para las mallas gruesa, media y fina son respectivamente: $10.693 \mathrm{KPa}$, $10.351 \mathrm{KPa}$ y $10.235 \mathrm{KPa}$, correspondientes a un rango de entre $6 \%$ y $2 \%$ de error frente al valor esperado de $\sigma_{f}=10 \mathrm{KPa}$.

Es remarcable la insensibilidad de la curva cargadesplazamiento al tipo de malla de elementos finitos utilizada. Esta «objetividad» de los resultados numéricos frente al tamaño de la malla del FEM es una de las características esenciales de la técnica FEM-DEM.

\subsection{Ensayo de cortante}

El ensayo de cortante se diseña para aplicar un esfuerzo de cizalladura sobre una probeta, de modo que experimente una falla por deslizamiento a lo largo de un plano paralelo a las 


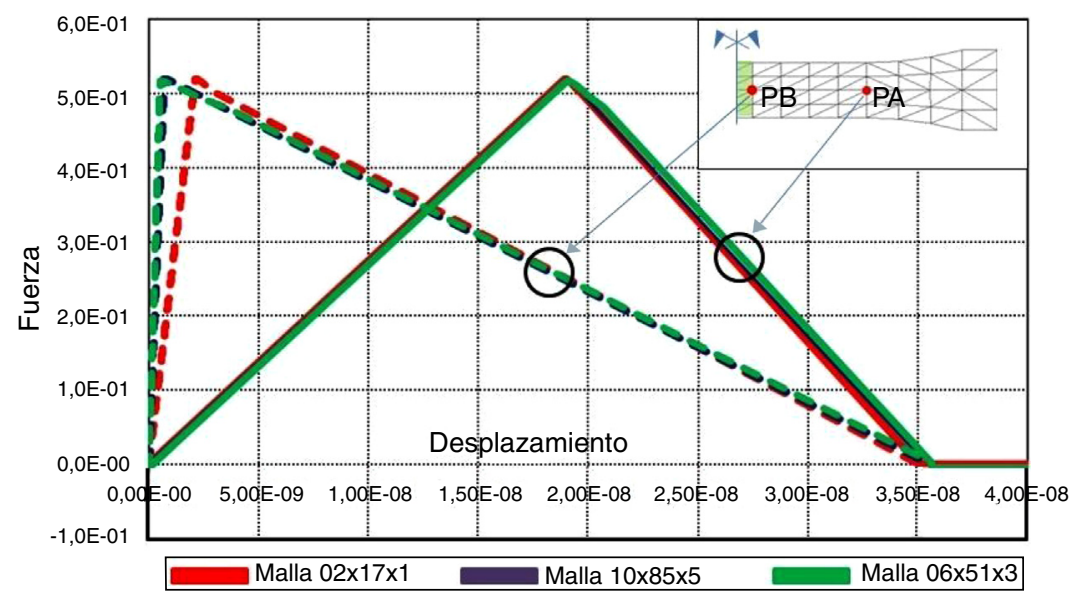

Figura 5. Ensayo normalizado 3D de tracción. Curva carga-desplazamiento de los puntos PA y PB de la muestra.

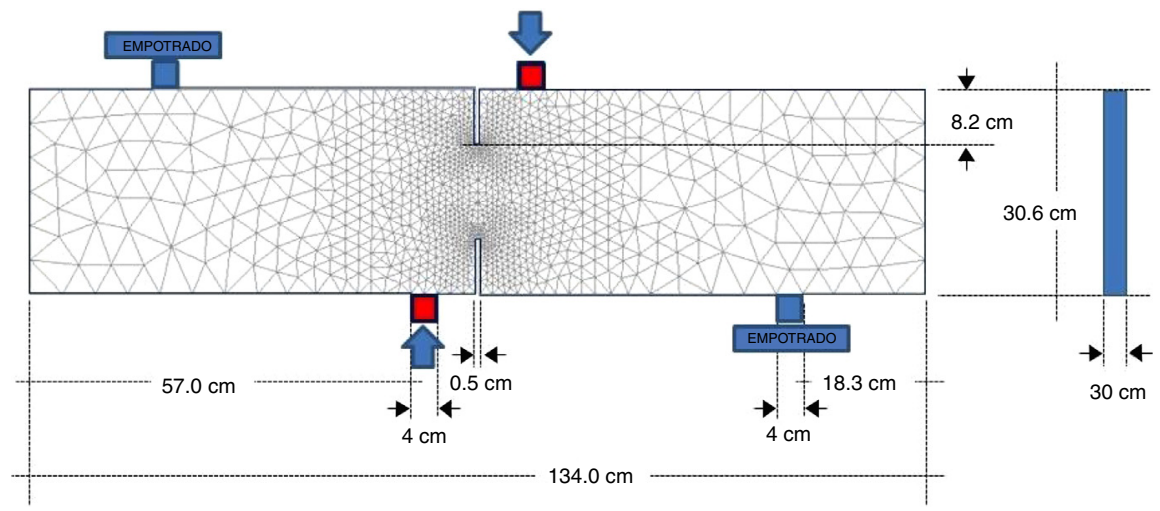

Figura 6. Viga bientallada a flexión. Geometría y condiciones de contorno.

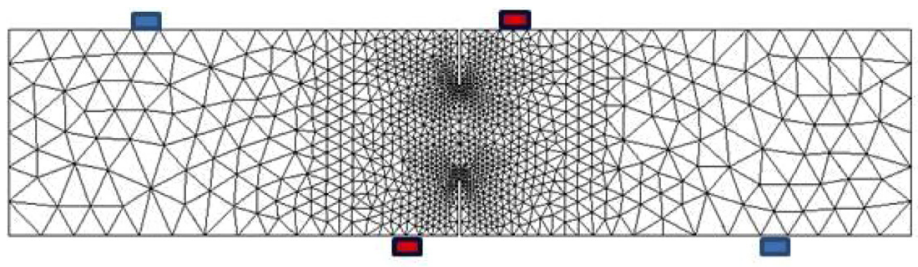

Malla gruesa

1165 nodos

2202 elementos

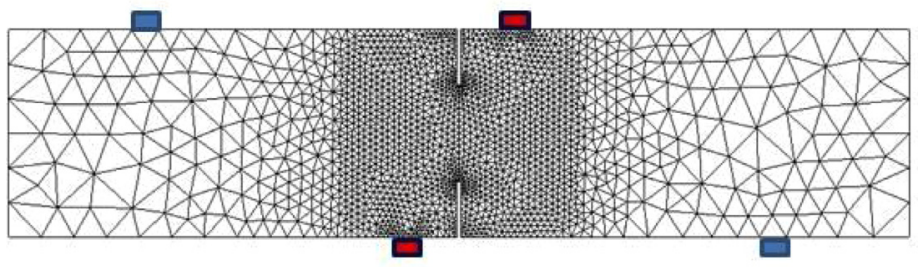

Malla media

1847 nodos 3480 elementos

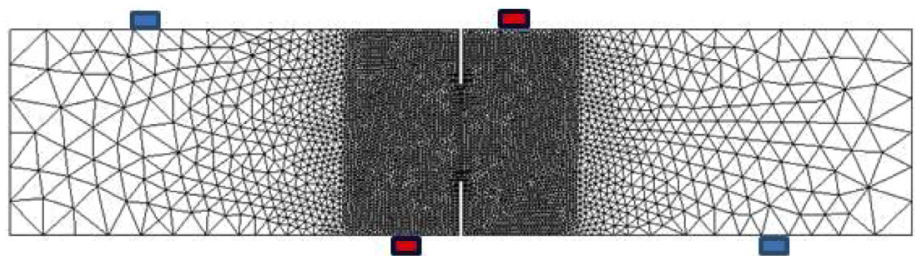

Malla fina

5747 nodos

11206 elementos

Figura 7. Viga bientallada a flexión. Mallas utilizadas. 


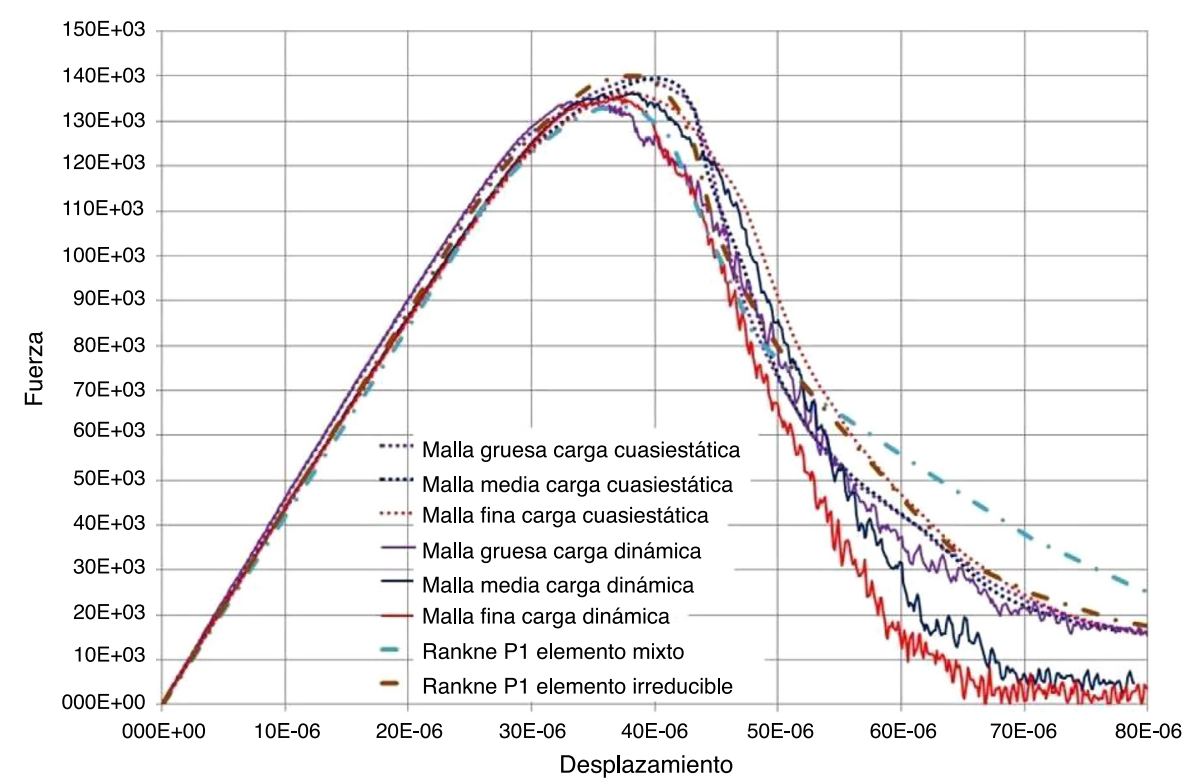

Figura 8. Viga bientallada a flexión. Relación entre la fuerza y el desplazamiento en cualquiera de los puntos referenciados en la figura 6. Los resultados obtenidos son comparados con aquellos dados en Cervera et al. [14].
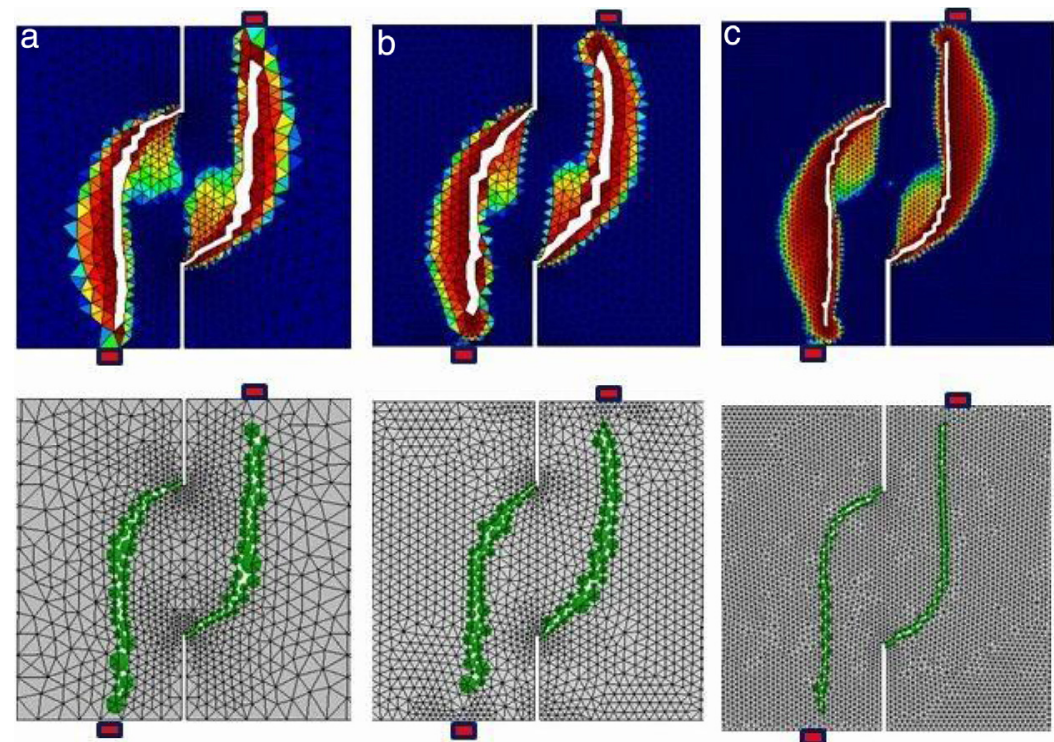

Figura 9. Viga bientallada a flexión. Superficies de igual desplazamiento y apertura de las grietas. a) Malla gruesa; b) malla intermedia; c) malla fina.

fuerzas aplicadas. Por lo general las fuerzas de cizallamiento provocan que una de las superficies de fallo de un material se mueva en una dirección y la otra superficie en dirección opuesta, de manera que el material se encuentra sometido a un estado de corte. Este ejemplo se realizó en 80 minutos usando un procesador a 2,5 MHz.

El ensayo que aquí se considera tiene como objetivo determinar la resistencia a cortante del hormigón y ha sido propuesto por Luong [21]. La probeta tiene forma tubular y su eje coincide con el eje $\mathrm{z}=0$. Tiene varias entallas y se somete a una carga central en una de sus caras y otra excéntrica en la cara opuesta, de manera que se generen tensiones cortantes paralelas al eje $\mathrm{z}=0$, como se describe en la figura 13 , en la que también se muestran las condiciones de contorno impuestas. La profundidad de la entalla es de $10 \mathrm{~mm}$ y su ancho es de $4 \mathrm{~mm}$. La carga se aplica imponiendo a la placa superior una velocidad constante de $1 \mathrm{~mm} / \mathrm{s}$ hasta llegar a la fractura.

La definición geométrica y la malla de elementos finitos utilizada se muestran en la figura 14. El plano de cizallamiento ha sido discretizado con 4 elementos finitos tetraédricos de 4 nodos, con el fin de captar adecuadamente el gradiente de tensiones en esta zona.

Las propiedades del hormigón son $E_{0}=35 \times 10^{9} \mathrm{~Pa}, v$ $=0,22, y=7,8 \times 10^{3} \mathrm{~N} / \mathrm{m}^{3}, \sigma_{c}=30 \mathrm{MPa}, \sigma_{f}=3 \mathrm{MPa}$ y $\mathrm{G}=75 \times 10^{-3} \mathrm{~J} / \mathrm{m}^{2}$. En general el esfuerzo máximo de corte 


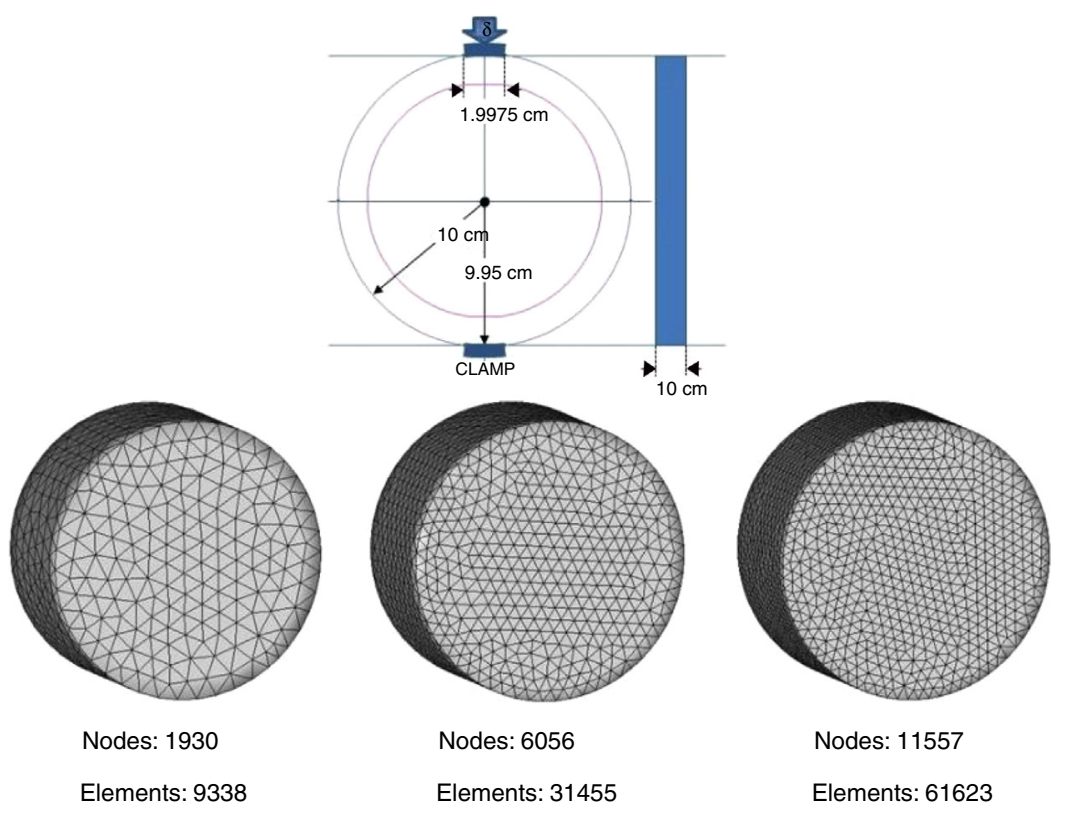

Figura 10. Ensayo de tracción indirecta. Dimensiones de la muestra, condiciones de contorno y mallas usadas de elementos finitos tetraédricos de 4 nodos.
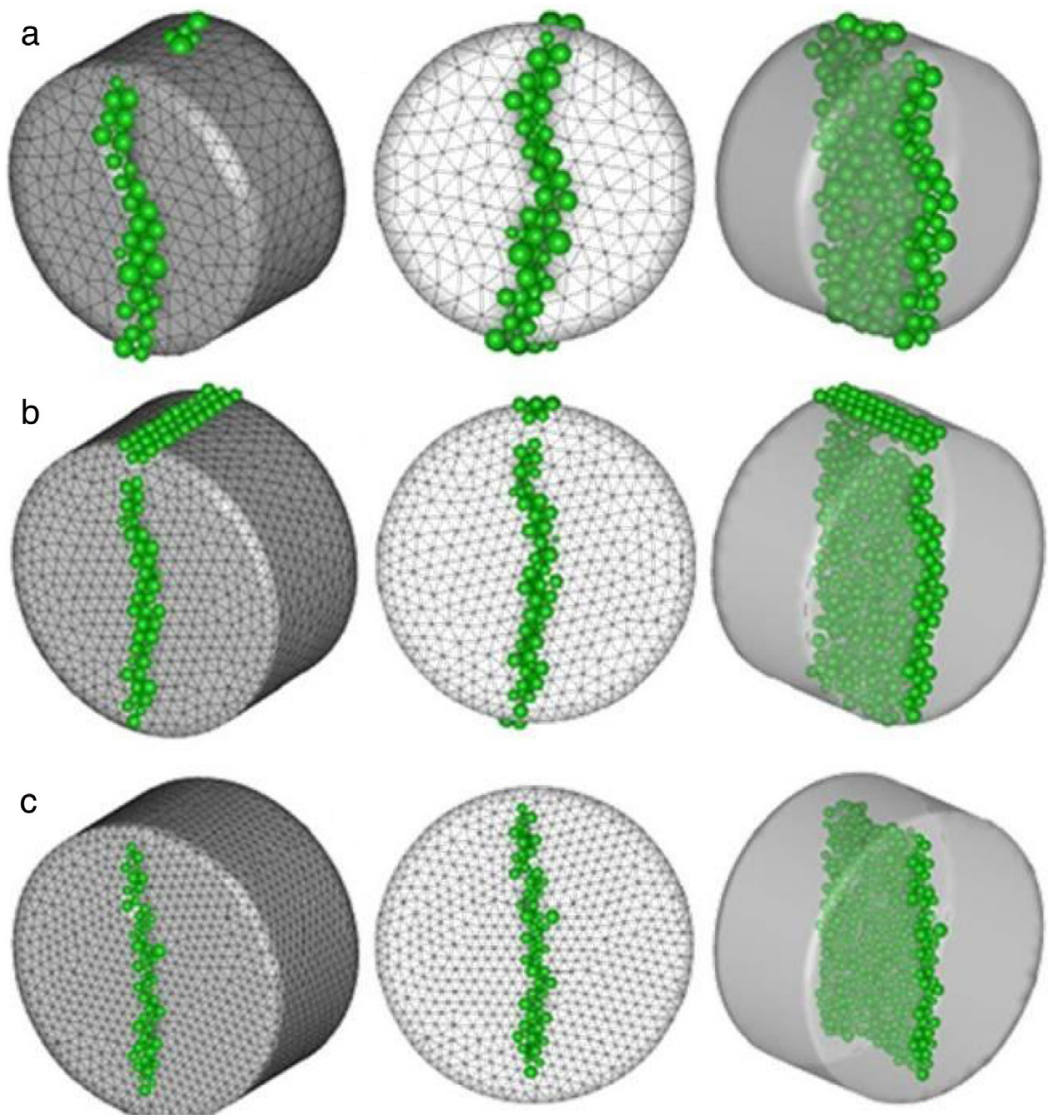

Figura 11. Ensayo de tracción indirecta. Grieta y elementos discretos generados. a) Malla gruesa; b) malla intermedia; c) malla fina.

en el hormigón es aproximadamente $1 / 5$ o $1 / 6$ del esfuerzo a compresión [21].

La figura 15 muestra la grieta obtenida por el experimento numérico con la técnica FEM-DEM, y se compara con los resultados de los ensayos realizados en el laboratorio.
La figura 16 muestra la relación fuerza-desplazamiento obtenida numéricamente y en la que se muestra claramente la rama elástica hasta que los elementos comienzan a dañar. Considerando el tamaño de la probeta, de esta gráfica se desprende que el esfuerzo cortante último alcanza un valor de 


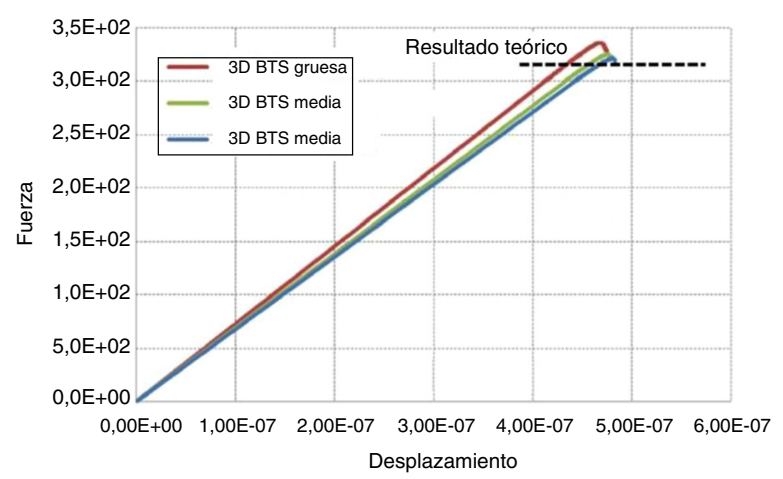

Figura 12. Ensayo de tracción indirecta. Relación fuerza-desplazamiento para las 3 mallas utilizadas.

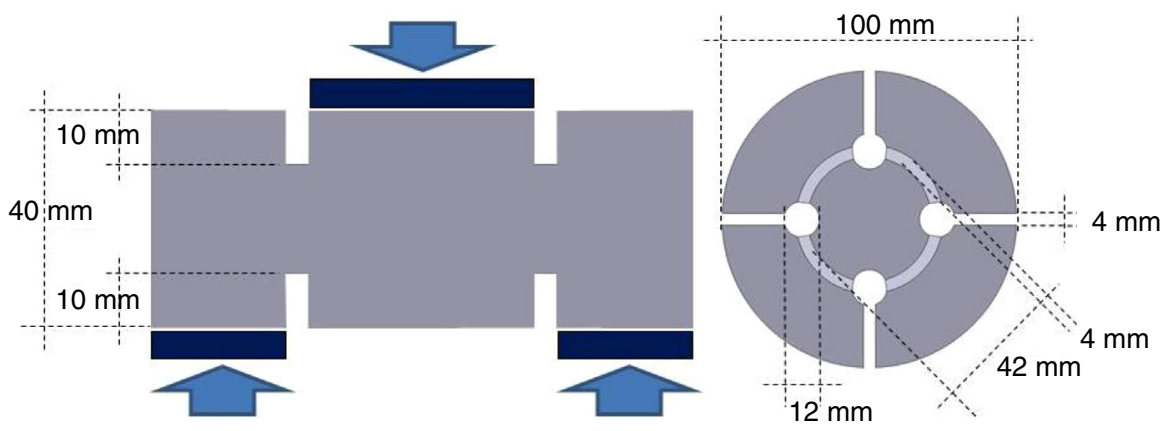

Figura 13. Ensayo de cortante. Geometría, cargas y condiciones de contorno.
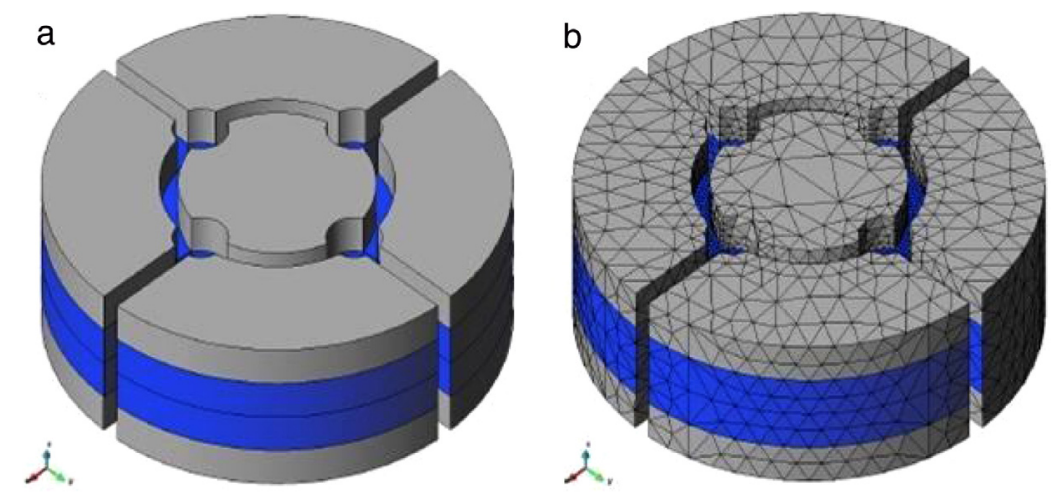

Figura 14. Ensayo de cortante. a) Definición geométrica de la probeta b) Malla de elementos finitos.

5,10 MPa. Este resultado es coherente y próximo al valor esperado [21].

\subsection{Ensayo de compresión simple}

Uno de los ensayos más frecuentes realizados en probetas de hormigón es el de compresión simple [25]. El ensayo se realiza de acuerdo con la instrucción de hormigón estructural (EHE-08) [26], la cual hace referencia a la norma UNE-EN 12390-3.2009 [27], en donde se especifica las dimensiones de las probetas y las condiciones de ensayo.

El experimento numérico que se presenta se realiza en 2D, utilizando probetas hexaédricas en tensión plana y para el caso 3D probetas cilíndricas normalizadas.
El ensayo 2D consiste en comprimir la probeta cuyas dimensiones y condiciones de carga se muestran en la figura 17 , y en la cual también se presentan las mallas utilizadas. El objetivo del ensayo no solo se trata de encontrar la tensión máxima a compresión que es capaz de resistir la probeta, sino también la forma de las fisuras en función de las distintas condiciones de contorno que se pueden dar en el ensayo.

Las propiedades del hormigón utilizadas son: $E_{0}$ $=30,0 \times 10^{9} \mathrm{~Pa}, v=0,20, y=24 \times 10^{3} \mathrm{~N} / \mathrm{m}^{3}, \sigma_{c}=2.000 \mathrm{MPa}$, $\sigma_{t}=20,0 \mathrm{MPa}$ y $\mathrm{G}=105 \times 10^{-3} \mathrm{~J} / \mathrm{m}^{2}$. En este caso la tensión máxima de compresión, independientemente de las condiciones de apoyo, es de $20 \times 10^{6} \mathrm{~Pa}$. En la figura 18 se muestra que los resultados obtenidos con ambas probetas se acercan mucho al resultado esperado. 

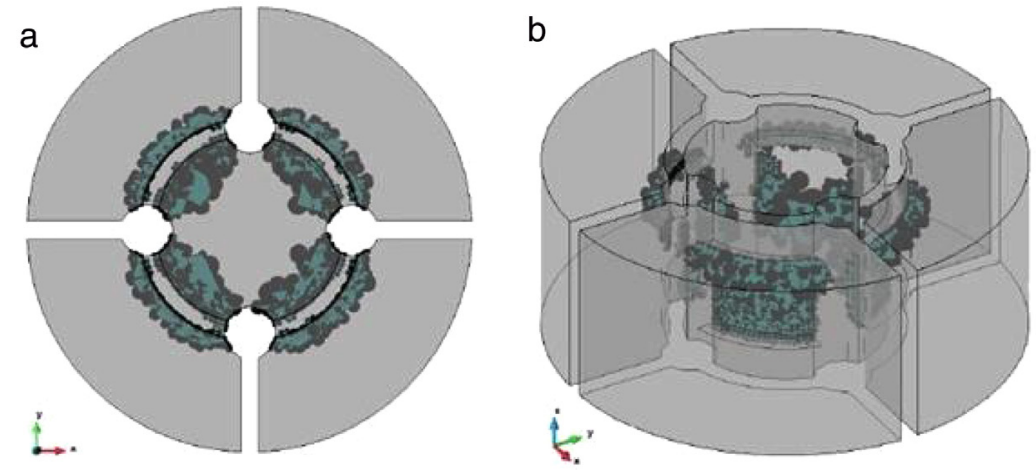

C

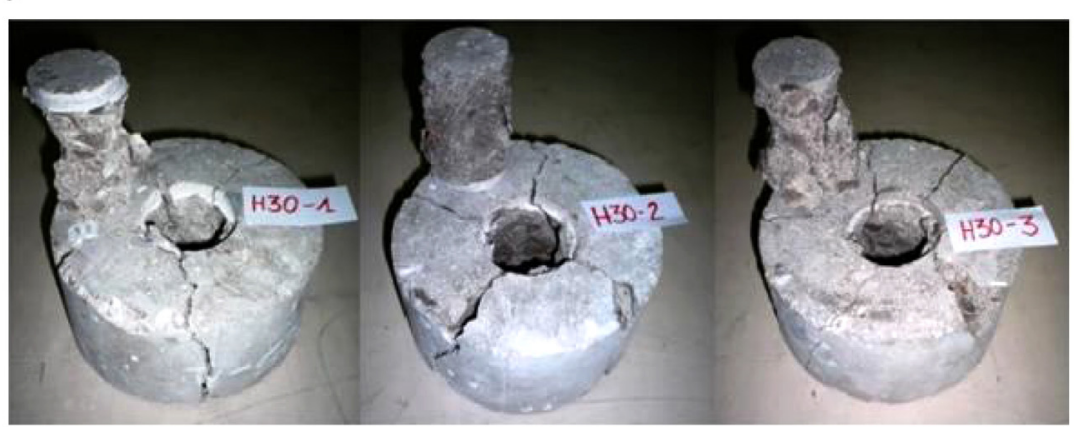

Figura 15. Ensayo de cortante. a) Vista superior; b) perspectiva de la grieta obtenida por la técnica FEM-DEM; c) rotura en probetas de laboratorio.

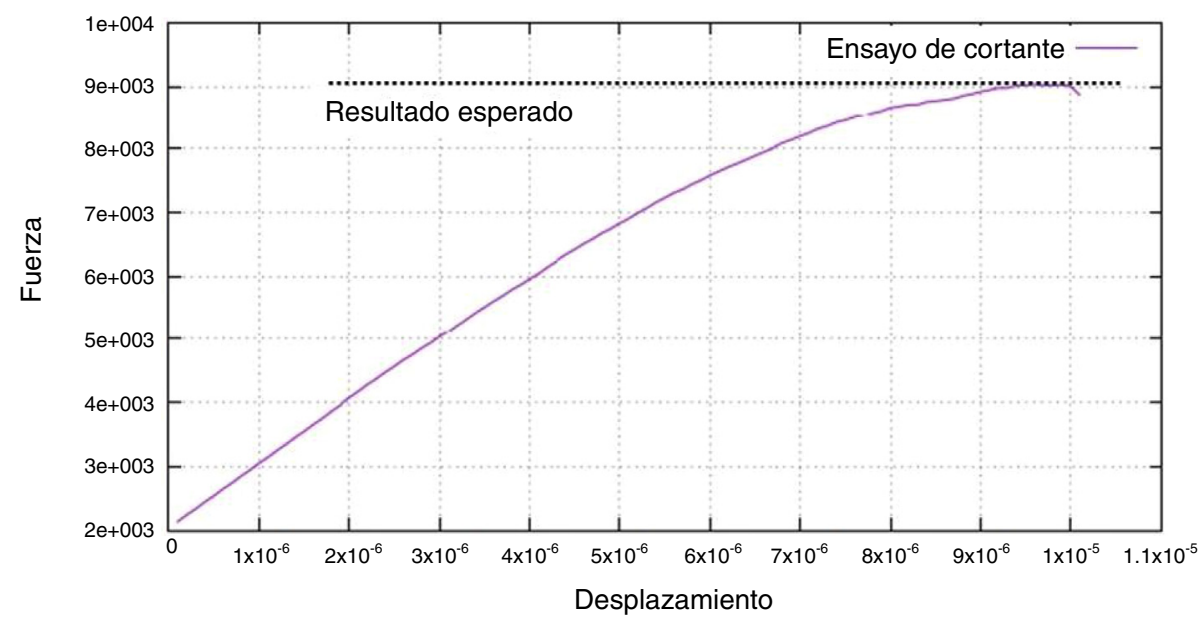

Figura 16. Ensayo de cortante. Relación fuerza-desplazamiento obtenida por el ensayo numérico.

Los resultados más interesantes en este caso se muestran en las figuras 19-21. En todas ellas la figura $a$ ) corresponde al patrón de grietas generado, la $b$ ) son las superficies de igual desplazamiento y la figura $c$ ) el daño elemental. Estos resultados se pueden comparar con la figura $d$ ) que corresponde a los resultados teóricos [28], o con la figura e), que corresponde a los resultados obtenidos con LS-DYNA [29] para el caso biempotrado (figs. 19 y 20) o con un ensayo real (fig. 21).

La figura 19 corresponde al caso en que las superficies de carga tienen un desplazamiento horizontal nulo utilizando la malla de elementos finitos estructurada. Los resultados muestran claramente 2 grietas a $45^{\circ}$ claramente definidas, y corroboradas por el patrón de daño mostrado. El resultado numérico mostrado en la figura $e$ ) muestra el mismo patrón. Sin embargo, observando los resultados teóricos [28] el daño se genera sobre 2 bandas a $45^{\circ}$ formadas por grietas verticales.

La figura 20 muestra el mismo caso, con el desplazamiento horizontal de las superficies de carga impedidas, pero utilizando la malla no estructurada. Como se puede observar en la fig. 20 a) el patrón de grietas se asemeja más a los resultados teóricos esperados, con grietas verticales localizadas en 2 bandas a $45^{\circ}$. Es importante mencionar que ningún código numérico al que los autores han tenido acceso es capaz de presentar este tipo de resultados, pues todos muestran correctamente la banda de daño (figuras e), pero no así las fisuras verticales sobre dicha banda. El por qué la malla no estructurada presenta un resultado más 


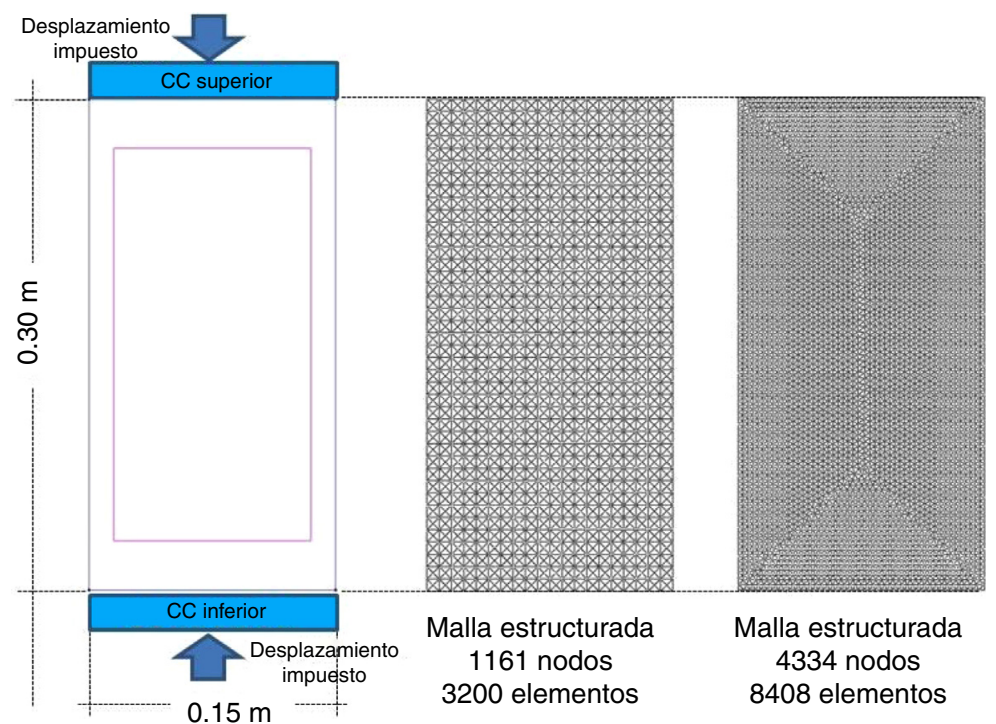

Figura 17. Ensayo de compresión simple. Probeta y mallas utilizadas en el ensayo 2D.

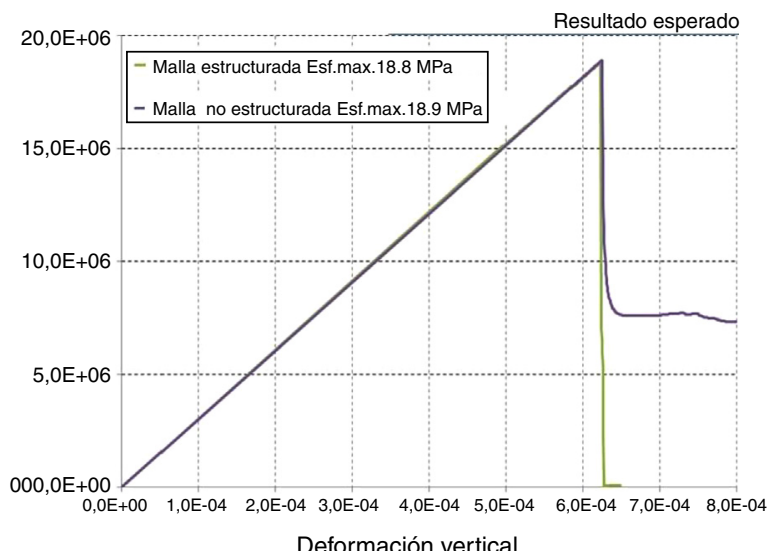

Figura 18. Ensayo de compresión simple. Relación tensión-deformación para el análisis 2D.

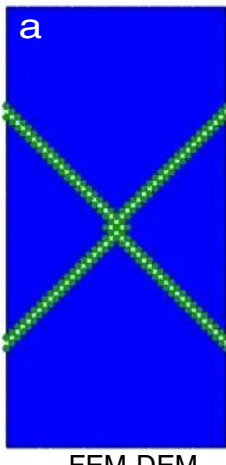

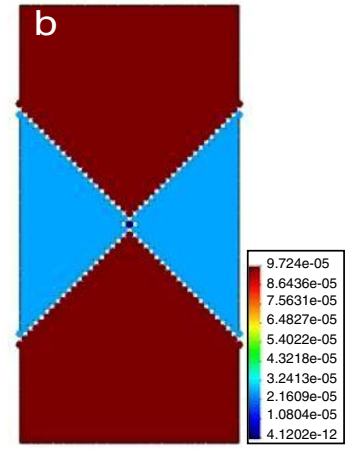

Desplazamientos

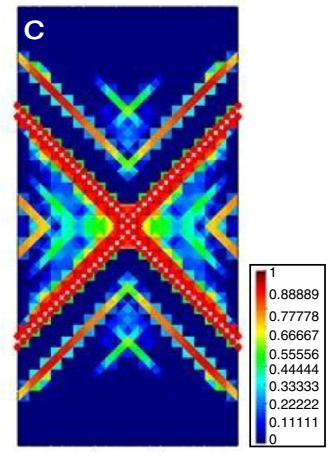

Daño

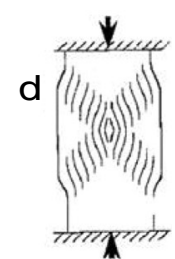

e

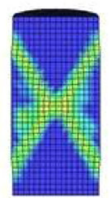

Figura 19. Ensayo de compresión simple. Probeta biempotrada, malla estructurada.

próximo al teórico está relacionado con la no uniformidad en la discretización de dominio. Las pequeñas variaciones numéricas inducidas por los elementos de distinto tamaño son suficientes para alterar la estabilidad de los resultados que se obtienen con una malla estructurada, encontrado así un resultado diferente.
La figura 21 presenta los resultados obtenidos con la malla no estructurada en el caso en que las superficies de carga tengan libertad de movimiento horizontal. Teóricamente [28], como se muestra en la figura $21 \mathrm{~d}$, la fisura debe ser vertical, sin embargo, como se observa en la figura 21 e los ensayos de laboratorio 


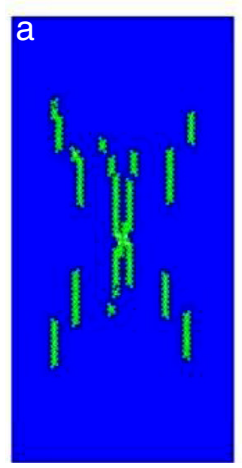

FEM-DEM

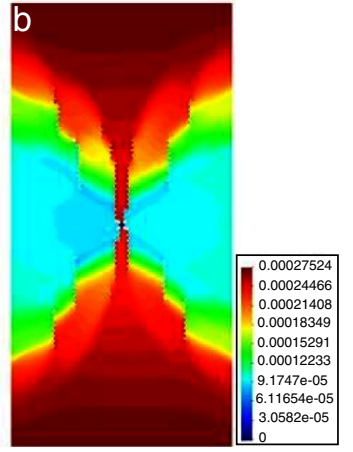

Desplazamientos

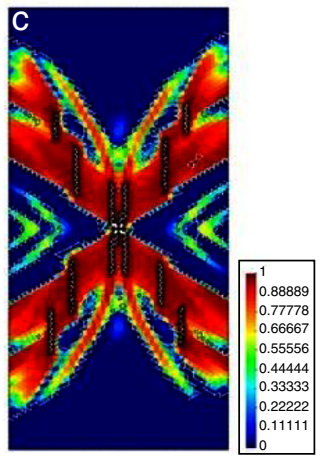

Daño

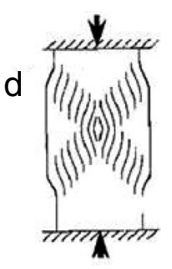

e

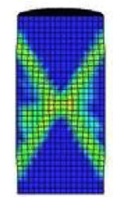

Figura 20. Ensayo de compresión simple. Probeta biempotrada, malla no estructurada.

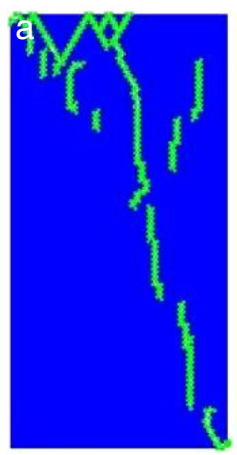

FEM-DEM

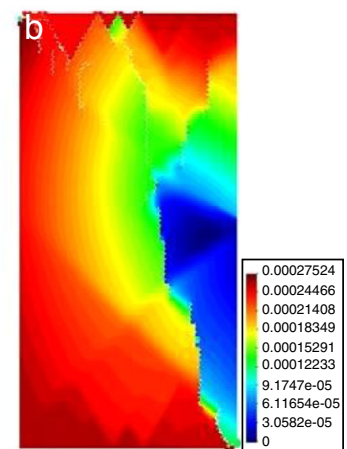

Desplazamientos

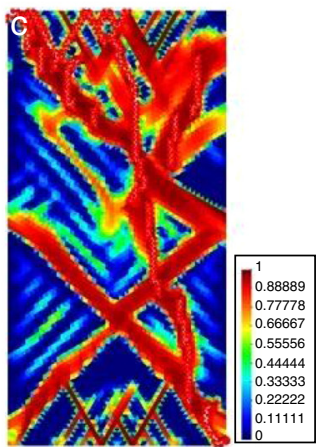

Daño

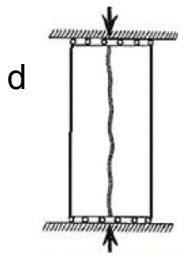

e

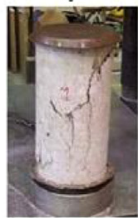

Figura 21. Ensayo de compresión simple. Probeta simplemente apoyada, malla no estructurada.
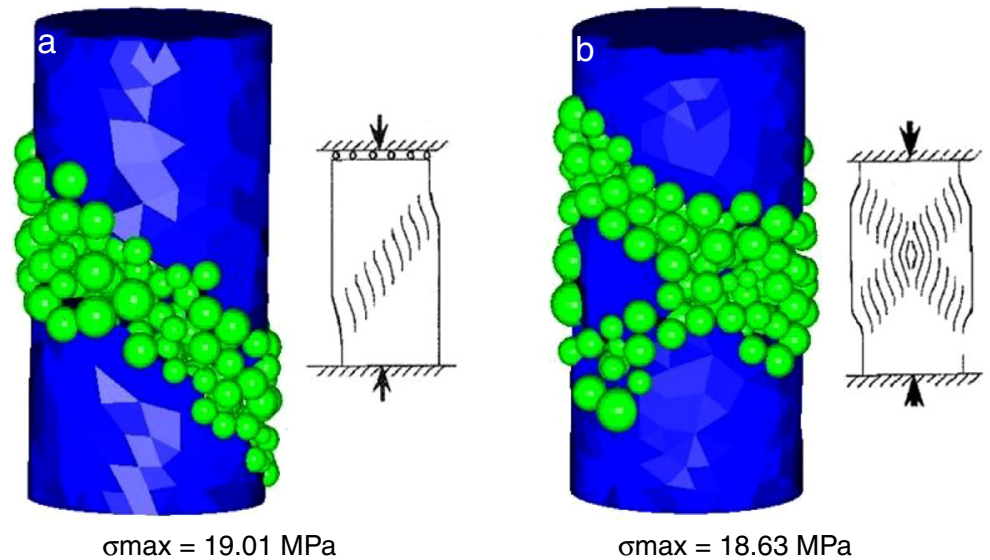

Figura 22. Ensayo de compresión simple. Probeta 3D para distintos casos de apoyo. a) Bases empotrada-libre; b) bases biempotradas.

tienden a presentar varias fisuras verticales [27]. Los resultados mostrados en la figura 21 a concuerdan con los resultados esperados.

El mismo ejemplo ha sido resuelto en 3D utilizando una probeta circular (fig. 17) y las mismas propiedades mecánicas. La malla está formada por 926 nodos y 4.030 tetraedros. Los resultados obtenidos se muestran en la figura 22 a para el caso en que una de las superficies de carga se encuentre impedida en su desplazamiento horizontal, mientras que la otra no. La figura $22 \mathrm{~b}$ muestra el caso en que ambas superficies tengan el desplazamiento horizontal impedido. En ambos ejemplos la tensión máxima se aproxima bastante a la esperada. No solo eso, a pesar que la malla sea tan gruesa, las grietas generadas coinciden con los resultados teóricos. Cabe comentar que este ejemplo no requiere más de 5 minutos de cálculo en un procesador a 2,5 GHz. 


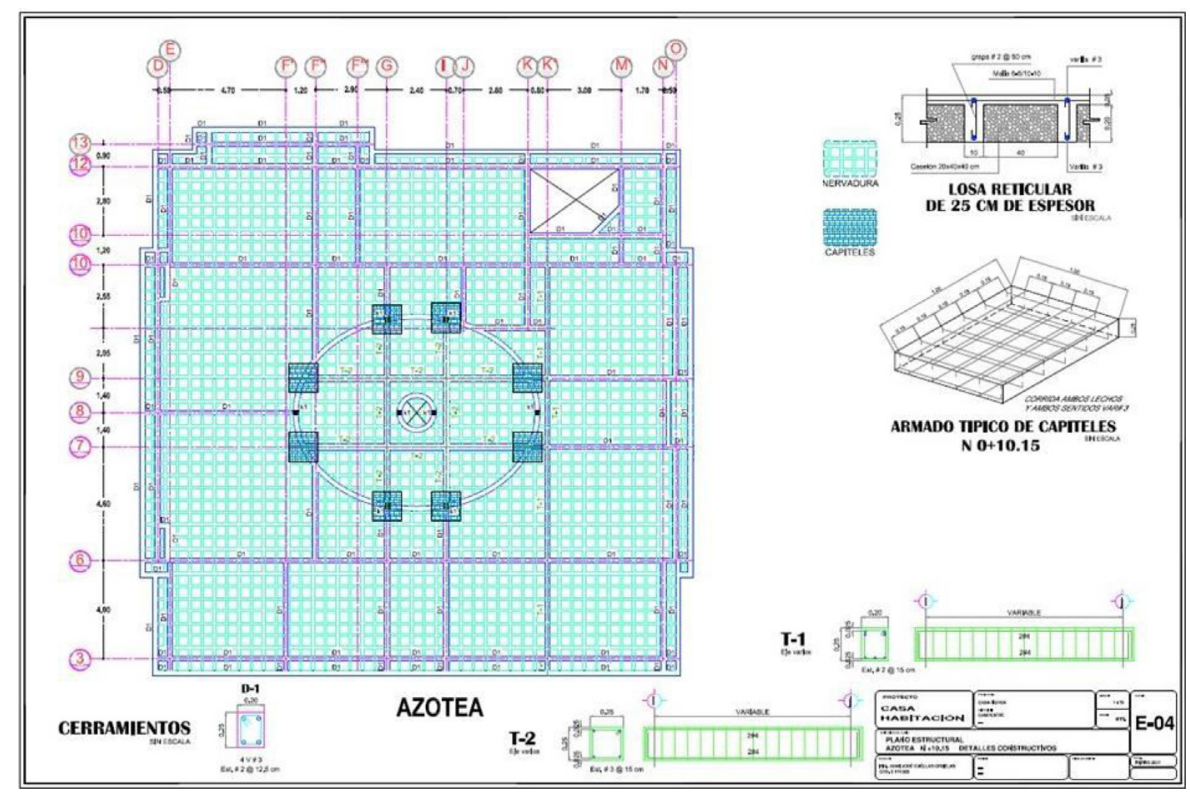

Figura 23. Losa reticular. Plano estructural.

\subsection{Losa reticular}

El uso de forjados reticulares como elementos estructurales está ampliamente consensuado y permite construir geometrías arquitectónicas más libres. El forjado analizado corresponde al techo de una vivienda. La planta es un cuadrado de $22 \mathrm{~m}$ de lado, con una superficie plana ovalada en el centro y los lados en parteaguas con un desnivel de $2 \mathrm{~m}$. En la figura 23 se muestra la planta estructural en donde se puede observar claramente la armadura de acero. Básicamente se trata de un forjado reticular de $0,25 \mathrm{~m}$ de espesor con una capa de compresión de $0,05 \mathrm{~m}$ y casetones de $0,40 \times 0,40 \times 0,20 \mathrm{~m}$. A lo largo de las nervaduras se colocan varillas de acero del $n .^{\circ} 3$ $(\phi=0,0095 \mathrm{~m})$.

Adicionalmente existe una serie de cerramientos (D1) y vigas (T1 y T2), así como 4 capiteles de columna. Los cerramientos tienen una sección de $0,25 \times 0,20 \mathrm{~m}$ armados con 4 varillas de acero del $\mathrm{n}$. $^{\circ}$. Las vigas T1 y T2 tienen un espesor de 0,25 m y un ancho de $0,20 \mathrm{~m}$ para la viga $\mathrm{T} 1 \mathrm{y}$ de $0,25 \mathrm{~m}$ para la viga T2. Ambas están armadas con 4 varillas de acero del n. ${ }^{\circ} 4$ $(\phi=0,0127 \mathrm{~m})$. Finalmente, el armado de los capiteles se realiza con varillas del n. 3 @ $0,15 \mathrm{~m}$ en ambos lechos y en ambas direcciones. La figura 24 a) muestra el armado de toda la losa, así como un detalle del armado de los capiteles en la figura 24 b).

Las propiedades del hormigón son. $E_{0}=21 \times 10^{9} \mathrm{~Pa}, v$ $=0,20, y=24 \times 10^{3} \mathrm{~N} / \mathrm{m}^{3}, \quad \sigma_{c}=20 \mathrm{MPa}, \sigma_{f}=2 \mathrm{MPa} y$ $\mathrm{G}=100 \times 10^{-3} \mathrm{~J} / \mathrm{m}^{2}$.

La placa se encuentra apoyada sobre los muros mostrados en la figura 25. A efectos del análisis dichos muros se consideran lo suficientemente rígidos y empotrados en su base.

Las cargas consideradas son el peso propio de la estructura, las cargas muertas $1.620 \mathrm{~N} / \mathrm{m}^{2}$ y las cargas vivas $400 \mathrm{~N} / \mathrm{m}^{2}$. Dichos valores consideran el peso del plafón, las instalaciones y acabados finales, sin considerar cargas de nieve o viento. Adicionalmente se impone un asentamiento diferencial de $0,0035 \mathrm{~m}$ de los puntos de la base del muro, como se indica en la figura 25.

El problema se ha resuelto utilizando una malla de 1.369,192 tetraedros y 368.969 nodos. Por otra parte, se han definido 5.084 elementos lineales para representar las varillas de acero. La figura 26 a representa el conjunto del forjado y muros a analizar, mientras que la figura 26 b corresponde a un detalle cercano al lucernario, donde se aprecian las nervaduras de la placa, así como algunas de las vigas $\mathrm{T} 2$.

La figura 27 a muestra los resultados del desplazamiento de la estructura después de que el asentamiento diferencial de la base sea de $0,0035 \mathrm{~m}$. En la figura $27 \mathrm{~b}$ se aprecia un corte muy definido en la cubierta superior. Se observa que el desgarro en la cubierta es perpendicular a la dirección del muro, poniendo de manifiesto la gran rigidez que tiene la cubierta superior.

En la figura 28 a se puede apreciar la deformidad de la estructura vista desde abajo. Es interesante observar que el lucernario sufre los desplazamientos máximos, incluso mayores a los que sufre el muro sujeto al asentamiento diferencial. La figura $28 \mathrm{~b}$ muestra una vista inferior de la losa donde se aprecia el daño producido en la unión del muro con esta.

En la figura 29 se observan los esfuerzos axiales sobre los elementos de acero pertenecientes a la viga $\mathrm{T} 2$ en la zona cercana al lucernario. En esta figura se puede observar los elementos en tracción y compresión debido a gran flexión a la que está sujeta esta parte de la estructura.

La figura 30 muestra las zonas dañadas de la estructura utilizando una visualización de la estructura deformada con un factor de escala de 5.000. El recuadro $a$ ) muestra una vista inferior de la losa en donde se puede observar la separación entre el muro y la losa, mientras que el recuadro $b$ ) muestra una vista superior mostrando el desgarro de la cubierta. En vista de los resultados obtenidos es claramente notoria la gran rigidez que posee la losa. 

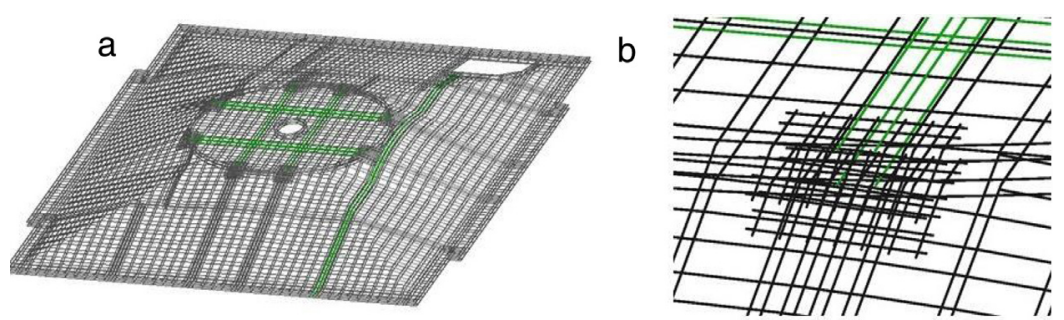

Figura 24. Losa reticular. a) Parrilla del armado de la losa; b) detalle del armado en los capiteles.

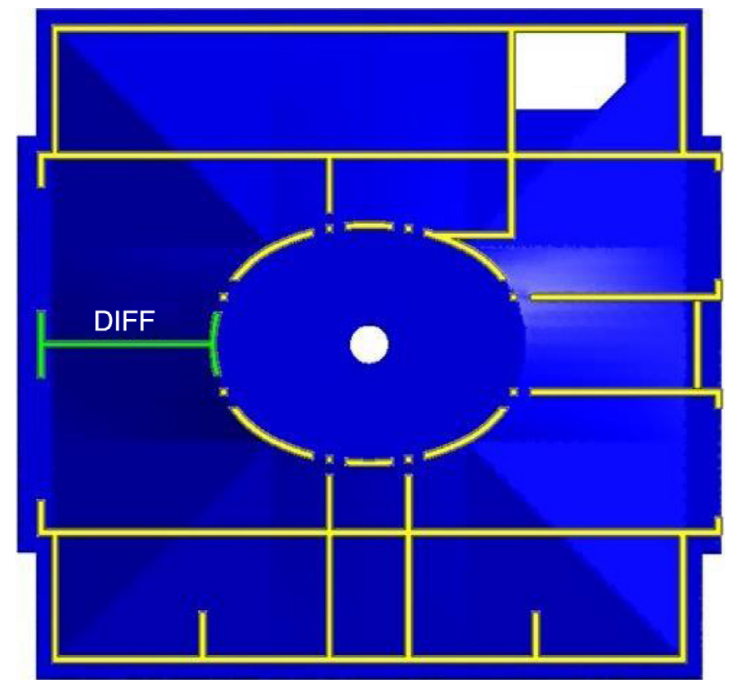

Figura 25. Losa reticular. Localización de los muros de carga. El muro con un asentamiento diferencial se encuentra marcado como DIFF.
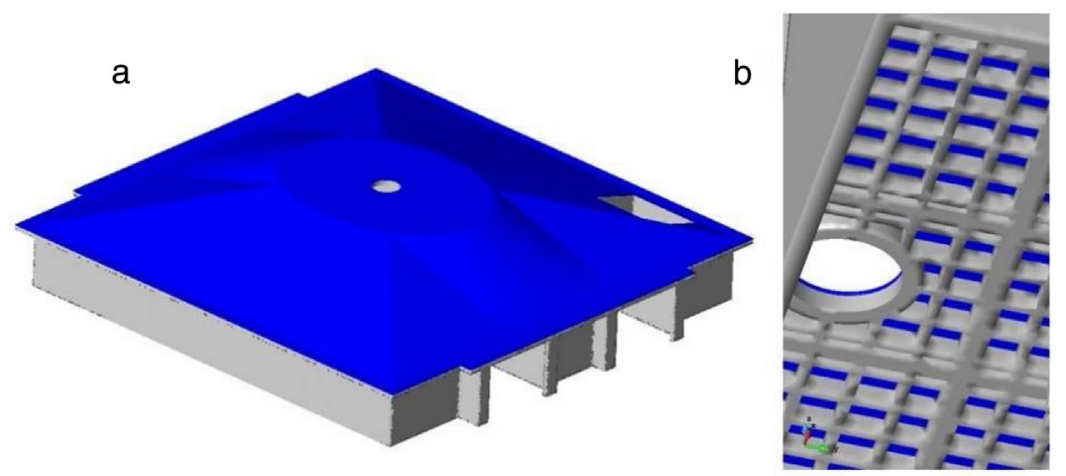

Figura 26. Losa reticular. a) Vista del conjunto; b) detalle de las nervaduras cercanas al lucernario.
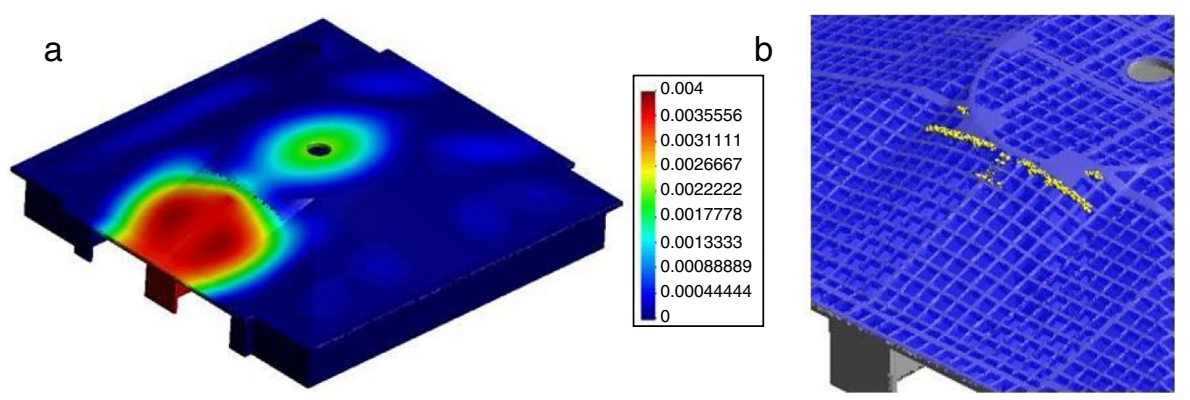

Figura 27. Losa reticular. a) Vista superior de la estructura con iso-superficies de igual desplazamiento; b) vista superior de las grietas generadas. 


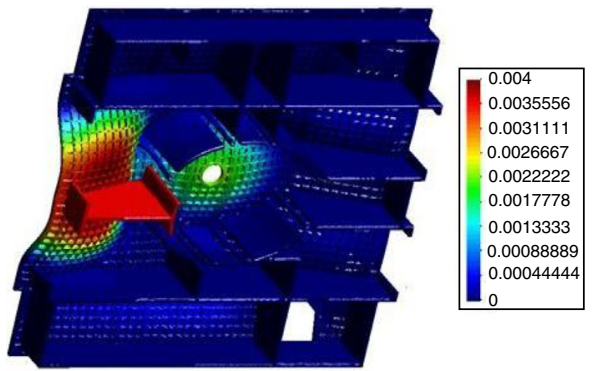

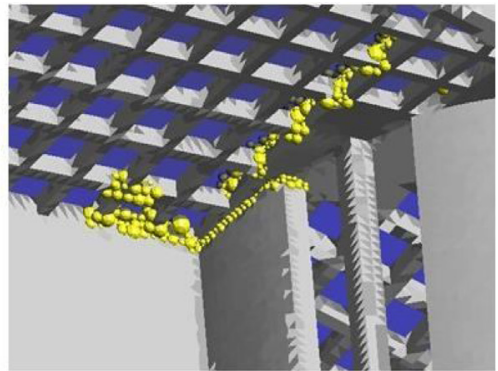

Figura 28. Losa reticular. a) Vista inferior de la estructura deformada; b) vista inferior de las grietas generadas.

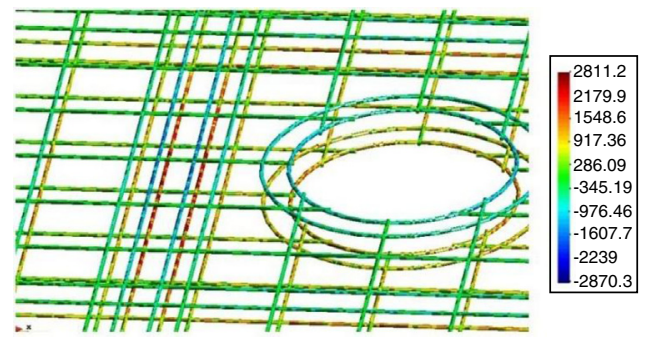

Figura 29. Losa reticular. Elementos del refuerzo de acero en la zona cercana al lucernario.
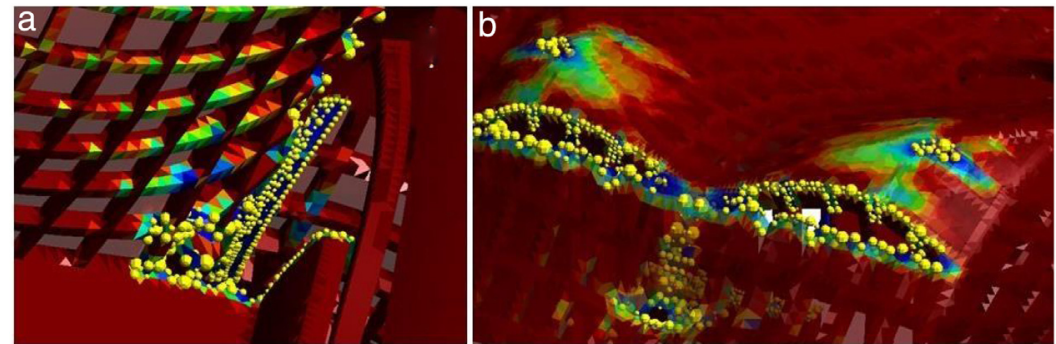

Figura 30. Losa reticular. Daño en la estructura. a) Vista inferior; b) vista superior.
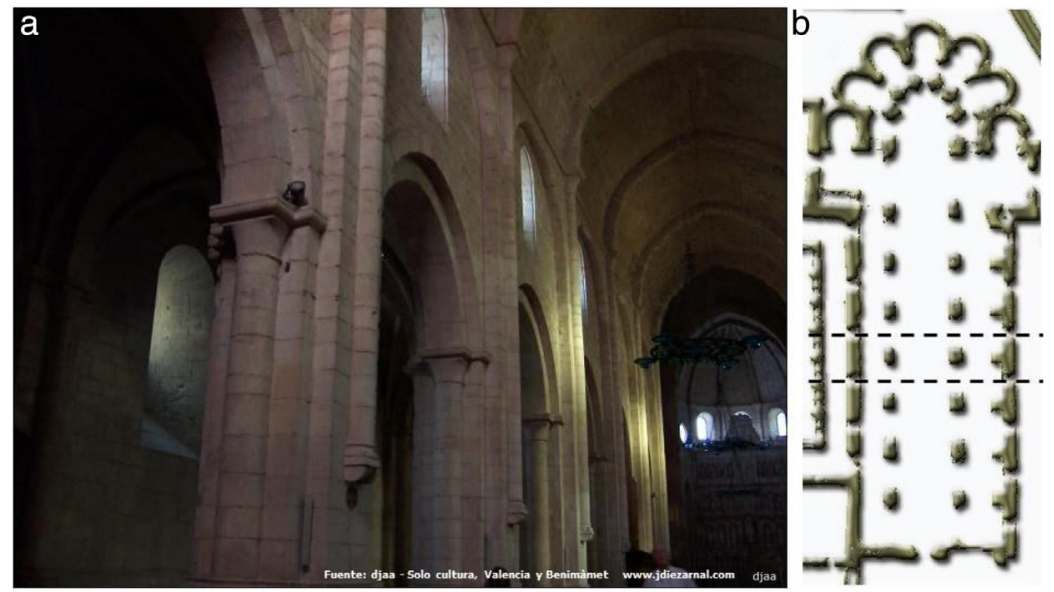

Figura 31. Iglesia cisterciense del monasterio de Poblet. a) Vista interior de la nave central; b) planta y sección analizada.

\subsection{Iglesia cisterciense del monasterio de Poblet}

La iglesia, perteneciente al Real Monasterio de Santa María de Poblet, ubicado en Vimbod (Tarragona), es un monumento histórico artístico declarado por la UNESCO como patrimonio de la humanidad. Construido a partir de la segunda mitad del siglo XII, el templo adopta una planta basilical, orientado su ábside al Este. La integran 3 naves de 7 tramos, con crucero, ábside central, girola y capillas absidiales. La nave central tiene unas dimensiones de $85 \mathrm{~m}$ de longitud, $21 \mathrm{~m}$ de anchura y $28 \mathrm{~m}$ de altura, mientras que las laterales alcanzan los $18 \mathrm{~m}$ de altura. La diferencia de altura respecto a la nave central no se resuelve 

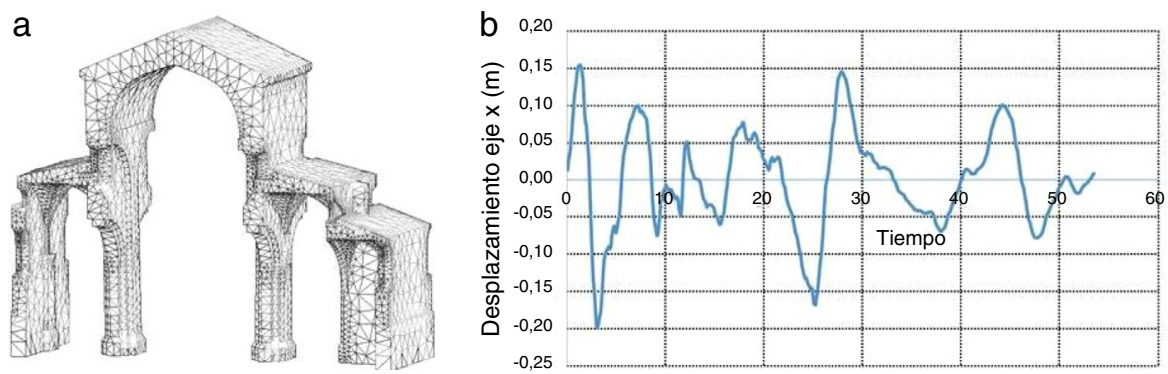

Figura 32. Iglesia cisterciense del monasterio de Poblet. a) Malla de elementos finitos de una sección perteneciente a la nave principal; b) excitación temporal en desplazamientos.

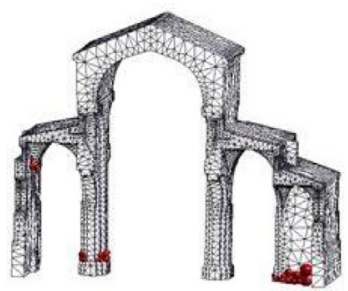

$\mathrm{t}=1.0 \mathrm{seg}$.

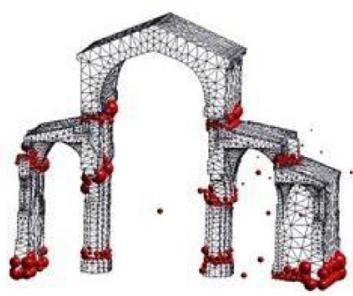

$\mathrm{t}=4.0 \mathrm{seg}$
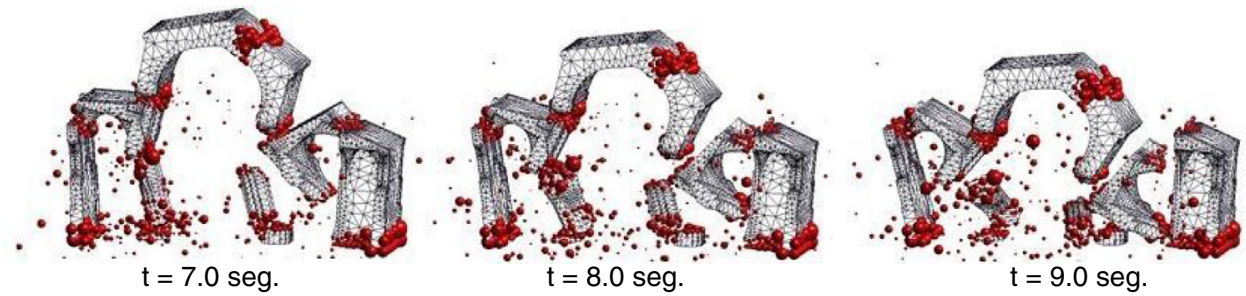

Figura 33. Iglesia cisterciense del monasterio de Poblet sometida a un terremoto. Evolución de las fracturas en la nave central en distintos tiempos.

mediante arbotantes del estilo gótico, sino con contrafuertes. La nave central plenamente románica está cubierta con bóveda de cañón apuntada, con arcos fajones en cada tramo, como se muestra en la figura 31.

Para simplificar el estudio se analiza únicamente una sección de la nave central que se considera simétrica respecto al eje normal a la dirección de dicha nave. La malla que discretiza la iglesia tiene 9.294 nodos y 32.487 elementos tetraédricos de 4 nodos, como se muestra en la figura $32 \mathrm{a}$. Las propiedades del material utilizado se corresponden con una piedra caliza propia de la región, con $E_{0}=35 \times 10^{9} \mathrm{~Pa}, v=0,22, y=25 \times 103 \mathrm{~N} / \mathrm{m}^{3}, \sigma_{c}$ $=780 \mathrm{MPa}, \sigma_{f}=5 \mathrm{MPa}$ y $\mathrm{G}=100 \times 10^{-3} \mathrm{~J} / \mathrm{m}^{2}$. En cuanto a las condiciones de apoyo se considera empotrada toda la superficie inferior del modelo.

Las cargas aplicadas corresponden a un movimiento oscilatorio impuesto en dirección normal al eje de la nave central, como se muestra en la figura $32 \mathrm{~b}$. Los valores mostrados corresponden al movimiento oscilatorio en dirección S 00E de los registros en desplazamientos obtenidos del acelerograma del sismo «El Centro» del 18 de mayo de 1940 [30]. Estos valores se aplican al modelo con una reducción del $50 \%$, de forma que simule un sismo de magnitud $6,0 \mathrm{M}_{\mathrm{W}}$.

Aunque el registro de movimientos dura más de 50 segundos la estructura solo es capaz de soportar en pie 4 segundos, colapsando de forma total a partir de ese instante. En la figura 33 se observa a intervalos de un segundo las grietas formadas en la iglesia. Inicialmente ocurre un cizallamiento de las columnas y de la pared más rígida, para posteriormente cortar las bases de los arcos. A partir del segundo 5 se observa cómo la estructura colapsa en su totalidad reduciéndose a escombros.

Este ejemplo muestra claramente la capacidad de la técnica FEM-DEM para simular la aparición y propagación de múltiples fracturas en estructuras de mampostería, así como el eventual colapso de la estructura bajo cargas dinámicas. 


\section{Conclusiones}

Se ha descrito en el presente artículo las líneas generales de la metodología FEM-DEM propuesta por los autores en Zárate y Oñate [9] y Zárate et al. [10] para la predicción de la aparición y evolución de fisuras en estructuras de hormigón. Los ejemplos que se han presentado muestran las posibilidades de la técnica FEM-DEM para el cálculo no lineal de estructuras de hormigón en masa y armado, así como en estructuras de mampostería. La técnica FEM-DEM es también aplicable al estudio de la fractura en macizos rocosos [31,32].

\section{Agradecimientos}

Los autores agradecen la colaboración del Ing. Juan José Cuellar Ornelas, al facilitar de forma desinteresada la información usada en el análisis del forjado. Los resultados aquí presentados han sido obtenidos utilizando los programas FEM2DEM y DEMPACK (http.//www.cimne.com/dempack) en los que se ha implementado la metodología FEM-DEM descrita.

\section{Bibliografía}

[1] P.A. Cundall, O.D.L. Strack, A discrete numerical model for granular assemblies, Geotechnique 29 (1979) 47-65.

[2] Labra C. Advances in the development of the discrete element method for excavation processes, Ph.D. Thesis. Barcelona; 2012.

[3] J. Rojek, E. Oñate, F. Zarate, J. Miquel. Modelling of rock, soil and granular materials using spherical elements. Cracow: 2nd European Conference on Computational Mechanics ECCM-2001; 26-29 June 2001.

[4] J.R. Williams, O'Connor, discrete element simulation and contact problem, Archives of Computer Methods in Engineering 6 (1999) 279-304

[5] E. Oñate, F. Zárate, J. Miquel, M. Santasusana, M.A. Celigueta, F. Arrufat, et al., A local constitutive model for the discrete element method, Application to Geomaterials and Concrete, Computational Particle Mechanics 2 (2015) 139-160.

[6] S. Katagiri, S. Takada. Development of fem-dem combined method for fracture analysis of a continuos media. Memoirs of the Graduate School of Science and Technology. Kobe University Japan; 20A (2002-03), 65-79.

[7] A. Munjiza. The combined finite-discrete element method. John Wiley \& Sons Ltd, England; 2004.

[8] S. Shmauder, J. Wulf, H.F. Fischmeister, Finite element modelling of crack propagation in ductile fracture, Computation Materials Science 1 (1993) 297-301.

[9] F. Zárate, E. Oñate, A simple FEM-DEM technique for fracture prediction in materials and structures, Computational Particle Mechanics 2 (2015) 301-314.

[10] F. Zárate, A. Cornejo, E. Oñate, A three-dimensional FEM-DEM technique for predicting the evolution of fracture in geomaterials and concrete, Computational Particle Mechanics (2017), 10.1007/s40571-017z.0178.

[11] E. Oñate. Cálculo de estructuras por el método de elementos finitos. Análisis estático lineal. Vol. 1. Sólidos (CIMNE, Barcelona, 2017, en castellano). Vol 2. Placas y láminas (Springer, Barcelona, 2009, en inglés).

[12] M. Cervera, M. Chiumenti, C. Agelet de Saracibar, Shear band localization via local j2 continuum damage mechanics, Computer Methods in Applied Mechanics and Engineering 193 (2004) 849-880.
[13] M. Cervera, M. Chiumenti, R. Codina, Mixed stabilized finite element methods in nonlinear solid mechanic's part I. Formulation, Computer Methods in Applied Mechanics and Engineering 199 (2010) $2559-2570$

[14] M. Cervera, M. Chiumenti, R. Codina, Mesh objective modelling of cracks using continuous linear strain and displacements interpolations, International Journal for Numerical Methods in Engineering 87 (2011) 962-987.

[15] P.R. Johnson, N. Petrinic, E. Sli. Element -splitting for simulation of fracture in 3D solid continua. Barcelona: VIII International Conference on Computational Plasticity; 2005.

[16] L. Mishnaevsky Jr., N. Lippmann, S. Schmauder, Computational modelling of crack propagation in real microstructures of steels and virtual testing of artificially designed materials, International Journal of Fracture 120 (2003) $581-600$.

[17] J. Lopez, S. Oller, E. Oñate, J. Lubliner, A homogeneous constitutive model for masonry, International Journal for Numerical Methods in Engineering 46 (1999) 16511671.

[18] O.C. Zienkiewicz, J.Z. Zhu, The superconvergent patch recovery (spr) and adaptive finite element refinement, Computer Methods in Applied Mechanics and Engineering 101 (1992) 207-224.

[19] E. Oñate. Desarrollos y aplicaciones de modelos de fractura en la Escuela de Ingenieros de Caminos de Barcelona. Centro Internacional de Métodos Numéricos en Ingeniería, CIMNE; 2000.

[20] C. Labra, E. Oñate, High-density sphere packing for discrete element method simulations, Communications in Numerical Methods in Engineering 25 (2009) 837-849.

[21] M. Luong, Tensile and shear strengths of concrete and rock, Engineering Fracture Mechanics 1-3 (1990) 127-135.

[22] Astm standard d638-10, 2003. Standard test method for tensile properties of plastics. Astm international, west Conshohocken, PA, 2003. doi. 10.1520/d0638-10, www.astm.org.

[23] F. L. L. B. Carneiro. A new method to determine the tensile strength of concrete. Proceedings of the 5th meeting of the brazilian association for technical rules; 1943. p. 126-129 (Portuguese).

[24] C.G. Rocco, G.V. Guinea Tortuero, J. Planas Roselló, M. Elices Calafat, Efecto del tamaño de probeta sobre la resistencia a la tracción medida con el ensayo brasileño, Hormigón y Acero 204 (1997) 47-63.

[25] J. Rodríguez del Viso, J.R. Carmona, G. Ruiz López, Efecto de la forma y el tamaño de la probeta en la resistencia a compresión en hormigón de alta resistencia, Hormigón y Acero 248 (2008) 77-86.

[26] Norma a instrucción de hormigón estructural (EHE-08) [consultado 2017]. Disponible en: https//www.fomento.gob.es/MFOM/LANG_ CASTELLANO/ORGANOS_COLEGIADOS/MASORGANOS/CPH/ instrucciones/EHE_es/.

[27] UNE-EN 12390-3.2009 Ensayos de hormigón endurecido. Parte 3. Determinación de la resistencia a compresión de probetas (2009). Asociación Española de Normalización y Certificación AENOR.

[28] Z.P. Bazant, J. Planas, Fracture and size effect in concrete and other quasibrittle materials, CRC Press LLC, 1998.

[29] Y.D. Murray, A. Abu-Odeh, R. Bligh. Evaluation of LS-DYNA Concrete Material Model 159 Publication No. FHWA-HRT-05-063 (2007) US Department of Transportation.

[30] Ground accelerogram from el centro, imperial valley irrigation district (comp s00e) [consultado 2017]. Disponible en: http//www.eng. ucy.ac.cy/petros/earthquakes/eq1.txt.

[31] J.M. González, F. Zárate, E. Oñate, Pulse fracture simulation in shale rock reservoirs: DEM and FEM-DEM approaches, Computational Particle Mechanics 5 (2017) 355-373

[32] E. Oñate, F. Zárate, M.A. Celigueta, J.M. González, J. Miquel, J.M. Carbonell, et al., Advances in the DEM and coupled DEM and FEM techniques in non linear solid mechanics advances in computational plasticity, Springer, 2018, pp. 309-335. 